\section{APRIL 1977 \\ HEATING TOKAMAKS VIA THE ION-CYCLOTRON AND \\ ION-ION HYBRID RESONANCES}

BY

F. W. PERKINS

\section{PLASMA PHYSICS LABORATORY}

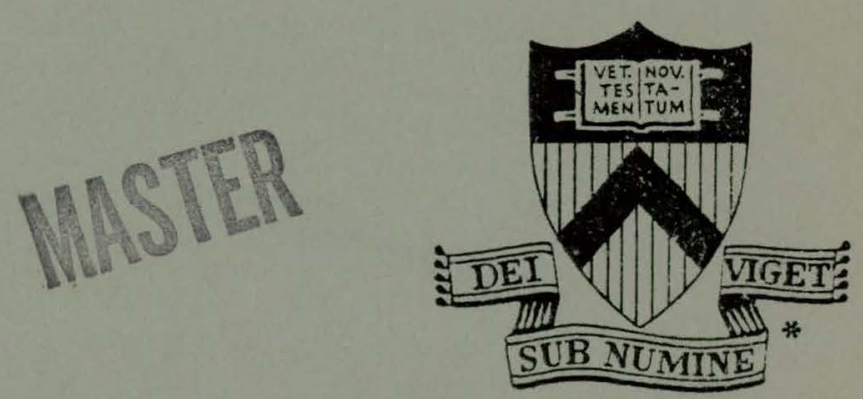

DISTRIEUTNN OF THIS DOCLRINA IS UNEIMITE

This work was supported by U. S. Energy Research and Development Administration Contract $\mathrm{E}(11-1)-3073$. Reproduction, translation, publication, use and disposal, in whole or in part, by or for the Unitad States Government is permitted. 


\section{DISCLAIMER}

This report was prepared as an account of work sponsored by an agency of the United States Government. Neither the United States Government nor any agency Thereof, nor any of their employees, makes any warranty, express or implied, or assumes any legal liability or responsibility for the accuracy, completeness, or usefulness of any information, apparatus, product, or process disclosed, or represents that its use would not infringe privately owned rights. Reference herein to any specific commercial product, process, or service by trade name, trademark, manufacturer, or otherwise does not necessarily constitute or imply its endorsement, recommendation, or favoring by the United States Government or any agency thereof. The views and opinions of authors expressed herein do not necessarily state or reflect those of the United States Government or any agency thereof. 


\section{DISCLAIMER}

Portions of this document may be illegible in electronic image products. Images are produced from the best available original document. 
NOTICE

This report was prepared as an account of work sponsored by the United states Government. Neither the United States nor the United States Energy Research and Development Administration, nor any of their employees, nur any of their contractors, subcontractors, or their employees, makes any warranty, express or implied, or assumes any legal liability or responsibility for the accuracy, completeness or usefulness of any information, apparatus, product or process disclosed, or represents that its use would not infringe privately owned rights.

\section{Printed in the United States of America.}

$$
\text { Available from }
$$

National Technical Information Service

$U$. S. Department of Commerce 5285 Port Royal Road

Springfield, Virginia 22151

Price: Printed Copy \$_*; Microfiche $\$ 3.00$

*Pages

$$
1-50
$$

$51-150$

$151-325$

$326-500$

$501-1000$
NTIS

Selling Price

$\$ 4.00$

5.45

7.60

10.60

13.60 
Heating Tokamaks Via the Ion-Cyclotron and Ion-Ion Hybrid Resonances

\author{
F. W. Perkins * \\ Princeton Plasma Physics Laboratory \\ Princeton University, Princeton, N. J. 08540
}

PPPL-1336

April 1977

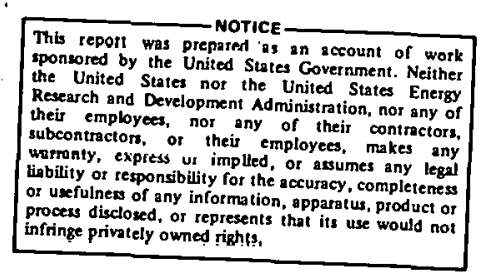

* Currently on leavc and working at National Center for Atmospheric Research

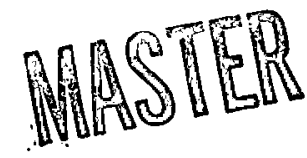

DISTRIBUTNUN OF THIS DOCLABENT IS UNHEMITER 
HEATING TOKAMAKS VIA THE ION-CYCLOTRON

AND ION-ION HYBRID RESONANCES

\section{F. W. Perkins*}

National Center for Atmospheric Research ${ }^{* *}$

Boulder, Colorado 80307

ABSTRACT. The ion-ion hybrid resonance can occur at high plarma densities in tokamaks and thereby absorb energy from the fast magnetosonic mode which would otherwise propagate freely. Ion-cyclotron resonance reglons, although they occur in the low density peripheral plasma, can nonetheless cause sufficient absorption to compete with fundamental cyclotron resonance damping by ions. For the ion-ion hybrid resonance it is shown that: (1) The energy absorption occurs via a sequence of mode converstons. (2) A poloidal field component normal to the ion-ion hybrid mode conversion surface strongly influences the mode conversion process so that rough ly equal electron and ion heating occurs in the present proton-deuterium experiments, while solely electron heating is predicted to prevail in deuteriumtritium reactors. (3) The ion-ion hybrid resonance suppresses toroidal eigenmodes. (4) Wave absorption in minority fundamental ion-cyclotron heating experiments will be dominated by ion-ion hybrid mode conversion absorption for minority concentrations exceeding roughly $1 \%$.

\footnotetext{
*On leave from the Plasma Physics Laboratory, Princeton University, Princeton, New Jersey 08540.

** Supported by the National Science Foundation.
} 
For the ion-cyclotron resonance, it is shown that: (1) Ion-cyclotron mode conversion leads to surface electron heating. (2) Ion-cyclotron mode conversion absorption dominates fundamental ion-cyclotron absorption thereby preventing efficient ion heating.

As a specific example, in "second harmonic heating experiments" of deuterium plasmas containing small concentrations of protons, wave absorption proceeds via the ion-ion hybrid mode conversion and the actual ion heating results froun cyclotron damping of the converted wave. True second harmonic heating in the PLT tokamak can be obtained by using a proton-Hc ${ }^{3}$ plasmá which has the same ratio of gyrofrequencles as a deuterium-tritium plasma and should accurately simulate reactor ion-cyclotron heating physics.

\section{INTRODUCTION}

The heating of tokamak plasmas has become one of the key objectives of controlled fusion research. Among the various paths towards this goal, heating via the fast magnetosonic mode in the Ion Cyclotron Range of Frequencies (ICRF) offers a variety of wave absorption processes which can bring aboul either electron or ion heating. This paper concentrates on wave absorption via the ion-ion hybrid and ion-cyclotron resonances.

Why is ion-ion hybrid absorption important? There are both experimental and theoretical reasons. Turning first to the experimental motivations, one notes that recent experiments on the ATC [1], TFR [2], and TM-1HF [3] tokamaks attempting secend harmonic heating of deuterium plasmas, have all uncovered similar phenomeña; unexpected on the basis of the theory of a single species plasma:(1) Toroidal eigenmodes either had a 
very low $Q$ or did not exist (see Fig. 6 of [2]). (2) The dependence of eigenmode amplitudes on the position of the $\omega=2 \omega_{C D}$ surface was not symmetric about the midline of the minor cross section. And (3), strong ion heating was observed. A single species model [4] predicts high-Q eigenmodes, symmetry of $Q$ for deviations of the $\omega=2 \omega_{c D}$ plane about the midline of the minor cross section, and weak ion heating (for a given wave field strength). A clear discrepancy between theory and experiment exists, and in view of the necessity to scale small tokamak results to reactor scale devices, it is important to obtain an understanding of why this discrepancy exists. The model invoked here is that the deuterium plasmas actually contained an important concentration of protons $\left(0.20 \geqslant n_{p} \geq 0.01\right)$ so that the proton-deuteron hybrid resonance surface lay close to the $\omega=2 \omega_{C D}=\omega_{C P}$ surface. Small concentratians of protons are indeed expected and measured [2] because of the operating procedures of the ATC and TFR experiments wherein deuterium heating experiments are interspersed among proton confinement experiments on a day-to-day schedule. One should note however, that TFR experiments on proton plasmas near the second harmonic of the proton gyrofrequency, where ion-ion hybrid effects do not play a role, have qualitatively verified the single species theory (Fig. 6 of [2]). True second harmonic heating of deuterium, uncontaminated by the ion-ion hybrid resonance and proton fundamental absorption, occurs only for proton concentrations less than $\beta_{i}$ - a demanding requirement experimentally.

From a theoretical point of view, one would like to understand both the magnitude and mechanism of ion-ion hybrid absorption so that whatever benefits it may have can be tested and exploited in the forthcoming ICRF experiments on PLT and TFR 6IJU. 
Ion-ion hybrid absorption has been recognized recently [5-7] as likely to play an important role in plasma heating via magnetosonic waves, and Swanson [7] has presented preliminary solutions to the Budden equation [8] governing propagation of a fast wave normally incident on an ion-ion hybrid resonance sürface. This paper sets forth in more detail the geometry of the resonance surface in the minor cross-section surface, generalizes the fast wave propagation equation to arbitrary angle of incidence, and examines the fate of energy launched into the short wavelength modes by the conversion processes. The component of the poloidal field normal to the mode conversion layer (i.e., in the major radius direction) produces important effects in the mode conversion process.

The next section of the paper presents an overview of the models which we will use in reaching an understanding of ion-ion hybrid resonance effects. The paper then proceeds from a simplified cold plasma dispersion relation to warm plasma dispersion relations and several full wave solutions, including Budden model effects on toroidal eigenmodes. The concluding section discusses tokamak applications, including the suggestion of using $\mathrm{p}-\mathrm{He}^{3}$ plasmas. The gyrofrequency ratio for $\mathrm{p}-\mathrm{He}^{3}$ is the same as $\mathrm{D}-\mathrm{T}$, and should accurately simulate a reactior as well as allow pure second harmonic heating uncontaminated by ion-ion hybrid resonance effects:

\section{MODELS}

Our modél is best presented $\frac{1 n}{11}$ several stages, because in fact a variety of. models are employed to deal with the various geometries which the ion-ion hybrid resonance givẹs r rise to. Let us start with an overview of how our models develop. First, we use a W.K.B. ideal model to establish 
the frequencies and geometry of the various ideal resonances and cutoffs in a two-species plasma. The term "ideal model" means one in which the parallel electric field is neglected and cold plasma theory governs the perpendicular currents. The ideal model shows that ion-ion hybrid resonance effects occur in a layer which is thin compared to the minor radius so that the mode conversion process can be correctly described by a slab model in which the density and poloidal field remain fixed and the only spatial variation of equilibrium parameters is the change of magnetic field strength with major radius. Given this geometry, a full W.K.B. dispersion relation is developed which includes: (1) finite ion Larmor radius associated with both fundamental and second harmonic gyrofrequencies; (2) parallel electric fields; (3) poloidal field effects on $k_{11}$, and (4) an arbitrary ratio between the parallel phase velocity and electron thermal velocity. This W.K.B. dispersion relation allows us to identify what are the important physical processes to include in full wave solutions, as well as to estimate both the size of the mode conversion region, and the damping which the converted waves will suffer.

The next step is to carry out simplified full wave calculations corresponding to both the ideal and mode conversion W.K.B. dispersion relations. As has been the experience with other mode conversion processes, we prove that the total dissipation predicted by an ideal Budden model agrees with the more sophisticated mode conversion calculations provided there is no energy propagating towards the mode conversion in the short wavelength branches. With this expected result in hand, we return to the ideal model to find the effect of ion-ion hybrid resonance on toroidal eigenmodes. Throughout these calculations we concentrate on two special cases: (A) Minority 
protons in a deuterium plasma. In this case, deuterium second harmonic waves can exist in the ion-ion hyḅrid resonance region. (B) D-T (or $\mathrm{p}-\mathrm{He}^{3}$ ) plasmas with an emphasis on minority tritons in a deuterium plasma. In this case, there is no degeneracy between a second harmonic frequency and the ion-ion hybrid frequency.

\section{THE IDEAL MODET: CUTOFFS AND RESONANCES}

The simplest model of wave propagation in a tokamak is the ideal, W.K.B. model where only cold plasma motions are allowed and the parallel electric field is ignored. Furthermore in tokamak geometries when the poloidal magnetic field is weak compared to the toroidal field, the parallel wave number is quite accurately given by

$k_{\prime \prime}=\frac{n}{R} \approx$ constant

unless the perpendicular wave number becomes very large because of mode conversion processes. Hence within the ideal model, waves propagate with constant angular frequency $\omega$ and constant $k_{11}$. Assuming that the static magnetic field lies in the $z$-direction and that the wave propagates in the $x-z$ plane, the familiar technifiques of cold plasma theory yield the equations

$$
\begin{aligned}
& \left(A-k_{11}^{2}\right) E_{x}+i D E_{y}=0 \\
& -i D E_{x}+\left(A-k_{11}^{2}-k_{\perp}^{2}\right) E_{y} \equiv 0
\end{aligned}
$$


and the dispersion relation

$k_{\perp}^{2}\left(A-k_{11}^{2}\right)-\left(B-k_{11}^{2}\right)\left(C-k_{11}^{2}\right)=0$

where

$\mathrm{D}=\frac{\omega_{\mathrm{pD}}^{2}}{c^{2}} \sum_{\sigma} \cdot n_{\sigma}\left(\frac{2 z_{\sigma}}{\mathrm{A}_{\sigma}}\right) \frac{\omega^{3}}{\Omega_{\sigma}\left(\Omega_{\sigma}^{2}-\omega^{2}\right)}$

$A=\frac{\omega_{\mathrm{PD}}^{2}}{\mathrm{c}^{2}} \sum_{\sigma=\eta_{\sigma}}\left(\frac{2 \mathrm{I}_{\sigma}}{\mathrm{A}_{\sigma}}\right) \frac{\omega}{\Omega_{\sigma}^{2}-\omega^{2}}$

$B=A+D=\frac{\omega_{p D}^{2}}{c^{2}} \sum_{\sigma} \eta_{\sigma}\left(\frac{2 Z_{\sigma}}{A_{\sigma}}\right) \frac{\omega^{2}}{\Omega_{\sigma}\left(\Omega_{\sigma}-\omega\right)}$

$C=A-D=\frac{\dot{\omega}^{2}}{c^{2}} \sum_{\sigma} n_{\sigma}\left(\frac{2 z_{\sigma}}{A_{\sigma}}\right) \frac{\omega^{2}}{\left(\Omega_{\sigma}+(1)\right) \Omega_{\sigma}}$

with the definition

$n_{\sigma} \equiv \frac{n_{i \sigma} z_{i \sigma}}{n_{e}}=$ fraction of electrons contributed by species $\sigma$

$\Omega_{\sigma} \equiv z_{\sigma} e B / M_{\sigma} c$

$\omega_{\mathrm{p} D}^{2} \equiv 4 \pi e^{2} \mathrm{n}_{\mathrm{e}} / \mathrm{M}_{\mathrm{D}}=\begin{aligned} & \text { deuterium plasma frequency for } \\ & \text { full elecliun derisity }\end{aligned}$

Figure 1 portrays functions $A, B$ and $C$ for a D-T plasma. The qualitative behavior is the same. for other species.

Evidently, the surface where

$A-k_{11}^{2}=0$

defines the ion-ion hybrid resonance at which $k_{1}^{2} \rightarrow \infty$ according to the ideal model. Equation (11) also yields the single species ion-cyclotron 
resonances. The usual fast magnetosonic wave cutoff is given by

$C-k_{11}^{2}=0$

Formula (7) shows that this cutoff is not sensitive to plasma composition. The surface $B-k_{\prime \prime}^{2}=0$ is a cutoff that occurs near the ion-ion hybrid resonance producing the resonance-cutoff pair which the Budden equation describes. :

Figures $2=4$ plor the resonances and cutoffs for our special cases of interest, and show how these surfaces manifest themselves on the tokamak minor cross section. The actual plasma densities are given by

$n_{e}=4.1 \cdot 10^{12} \mathrm{~cm}^{-3}\left(\frac{1 \text { meter }}{\lambda_{11}}\right)^{2} \mathrm{~N}$

in terms of the quantity $\mathrm{N} \equiv \omega_{\mathrm{pD}}^{2} / \mathrm{c}^{2} \mathrm{k}_{11}^{2}$ read from Figures $2-4$. In using. Figs. 3 and 4 for a $\mathrm{p}^{-\mathrm{He}^{3}}$ plasma, the nondimensional frequency variable is $y=\omega / \Omega_{p}$ and the densities should be one-half those given by (13).

What physics does one learn from Figs. 2 - 4? First, it is evident that the geometry of the resonances and cutoffs is quite complicated, and prevents a simple one-dimensional model from describing the effects of the ion-ion hybrid resonance. Second, when the heavier inn has the sinaller concentration, ion-cyclotron mode conversion resonances exist over a good fraction of the plasma circumference, and compete with the ion-ion hybrid resonance. On the other hand, the mode conversion-cutoff regions are generally quite thin compared to the minor radius, pointing towards the use of a sequence of one dimensional models, each model treating the physics of the mode conversion process in a small region of space and connecting in a 
simplified way to global eigenmodes.

More specifically, in the case of present experiments, Fig. 2, with small concentrations of light ions (protons) in a heavy ion (deuterium) . plasma, the relative width in minor radius over which ion-ion hybrid effects occur is small. If one defines $\delta a$ to be the maximum deviation of an $A$ or $B$ surface from the $\omega=2 \Omega_{\mathrm{D}}$ surface, then for small proton concentrations one can rind

$\frac{\delta a}{a} \leqq \frac{3 R}{4 a} \eta_{p} \quad \frac{\delta a_{B}}{a} \leqq \frac{R}{2 a} \eta_{p}$

where the equalities apply at high densities. To the extent that

$\frac{\left|\delta a_{A}-\delta a_{B}\right|}{a} \approx \frac{R}{4 a} n_{p} \ll 1$,

the mode conversion layer is thin with respect to the minor radius and the specifics of the mode conversion processes can be obtained irom a onedimensional slab model with constant poloidal field and plasma density. Figurc $2 b$ also points out that it is impossible to avoid some ion-cyclotron mode conversion absorption in the tenuous plasma outside magnetosonic wave cutoff.

In the case of plasmas with a small concentration of heavier ions (tritons) in a light ion (deuterium) plasma, the presence of an ion-ion hybrid resonance surface is accompanied by two mode conversion-cutoff pairs corresponding to deuterium and triton ion-cyclotron waves. Again the layers will be thin in minor radius, and amenable to a slab type analysis. 
Thirdly, even in plasmas with $50 \%$ deuterium - 50\% tritium, the relative separation between the mode conversion resonance and cutoff is a small fraction of the minor radius. At high densities, one can show that

$\left|\delta a_{A}-\delta a_{B}\right| a^{-1} \approx 0.02(R / a)$

so that a slab type geometry is again a good model. In this case also, it is impossisiblé tó avold mode conversion absorption by ion-cyclotron resonances. Altogether, the complicated geometries depicted in Figs. 2 - 4 will prevent precise calculations of wave damping decrements, etc. The best this paper will do is factor-of-two estimates for the contributions of varinus processes to the overall wave damping decrements. But these estimates and the scaling laws associated with them form a sufficient understanding of the actual heating processes to be useful in guiding and interpreting tokamak heating experiments.

\section{FINLI'E LAKMUK KALIUS DISPE'KSIUN KELA'ILN}

The goal of this section is to determine what physical processes are playing a role in the mode conversion phenomena which occur when $k_{\underline{\perp}}^{2} \rightarrow \infty$ according to the ideal model [c.f. (3) and (10)]. Our tool for this will be a general finite Larmor radius difspersion relation and a one-dimensional slab geometry. Such an analysis produces three classes of results: (1) identification of the dominant terms governing the propagation of the short wavelength converted modes, (2) the principal damping processes for these modes, and (3), an estimate of the typical scale sizes of the mode conversion layer. 
The physics incorporated in the finite Larmor radius dispersion relation includes: (1) electron and ion velocities across the magnetic field according to cold plasma theory, (2) a Maxwellian velocity distribution for both electrons and ions, (3) the first finite Larmor radius contributions to ion velocities across the magnetic field which involve not only FLR corrections to the cold plasma velocities, but also the introduction of second harmonic terms, (4) arbitrary ratio of the parallel phase velocity of the wave to the electron thermal velocity, and (5) fundamental and second harmonic ion damping processes and electron Landon damping.

Figure 5 portrays our general one-dimensional slab model for investigating mode conversion physics. This model stems from the narrow spacing between the resonance and cutoff surfaces. Consequently, the equilibrium varies only in the $\mathrm{x}$-direction, while the $\mathrm{z}$-direction is the toroidal direction. The $\mathrm{y}$ direction completes a right-hand coordinate system.

The essence of our slab model is; that the $x$-direction is the local normal to the mode conversion surfaces portrayed in Figs. 2 - 4. Hence the actual orientation of the $\mathrm{x}$-direction in a laboratory frame depends on the mode conversion process under consideration. Let us discuss two extreme examples occurring in Fig. 3c. The ion-ion hybrid mode conversion region near the center of plasma is oriented so that the poloidal field is normal to the mode conversion layer, and the principal variation in the equilibrium results from the major radius variation of magnetic field intensity. On the other hand, the deuterium ion-cyclotron mode conversion surface on the outside of the plasma is oriented so that the poloidal field is parallel to the mode conversion surface, and it is the equilibrium density variation that induces the mode conversion process. In both these examples, the $x$-direction lies close to 
the major radius direction, but this will evidently not be the case for the deuterium ion-cyclotron mode conversion surfaces of Fig. $3 b$.

It is straightforward to obtain to the finite Larmor radius dispersion relation starting from the conductivity tensor $\overleftrightarrow{\sigma}$ on p. 229 of Bekefi's book [9] or Swanson's dielectric tenşor $\overleftrightarrow{\mathrm{K}}$ [10]. Let the wave-vector be given by

$\vec{k}=k_{1} \vec{e}_{1}+k_{11} \vec{e}_{3}$

and wave electric field amplitude by $\vec{E}(x, t)=\vec{E} \exp (i \vec{k} \cdot \vec{x}-i \omega t)$, so that the WKB wave equation becomes

$\overrightarrow{\mathrm{k}} \times(\overrightarrow{\mathrm{k}} \times \overrightarrow{\mathrm{E}})+\frac{4 \pi i \omega}{c^{2}} \overleftrightarrow{\sigma} \cdot \overrightarrow{\mathrm{E}}=0$

In component form, equation (18) is

$$
\begin{aligned}
& \left(\sum_{\sigma} A_{\sigma}-k_{11}^{2}\right) E_{1}+i\left(\sum_{\sigma} D_{\sigma}\right) E_{2}+k_{\perp} k_{11} E_{3}=0 \\
& -i\left(\sum_{\sigma} D_{\sigma}\right) E_{1}+\left(\sum_{\sigma} \bar{A}_{\sigma}-k_{\perp}^{2}-k_{11}^{2}\right) E_{2}=0 \\
& k_{\perp} k_{11} E_{1}+\left(\frac{\omega^{2}}{c^{2} k_{11}^{2} D_{e}^{2}} W_{e}-k_{\perp}^{2}\right) E_{3}=0
\end{aligned}
$$

where the subscript $\sigma$ denotes an iok species and

$$
A_{\sigma}=\frac{\omega_{\rho \sigma}^{2}}{c^{2}}\left[\frac{\omega^{2}}{\Omega_{\sigma}^{2}-\omega^{2}}\left(1-\frac{k_{\perp}^{2} T_{i}}{M_{\sigma} \Omega_{\sigma}^{2}}\right)+\frac{\omega^{2}}{4 \Omega_{\sigma}^{2}-\omega^{2}}\left(\frac{k_{\perp}^{2} T_{i}}{M_{\sigma} \Omega_{\sigma}^{2}}\right)\right]
$$




$$
\begin{aligned}
& \bar{A}_{\sigma}=\frac{\omega_{p \sigma}^{2}}{c^{2}}\left[\frac{\omega^{2}}{\Omega_{\sigma}^{2}-\omega^{2}}\left(1-\frac{3 k_{\perp}^{2} T_{i}}{M_{\sigma} \Omega_{\sigma}^{2}}\right)+\frac{\omega^{2}}{4 \Omega_{\sigma}^{2}-\omega^{2}}\left(\frac{k_{\perp}^{2} T_{i}}{M_{\sigma} \Omega_{\sigma}^{2}}\right)\right] \\
& D_{\sigma}=\frac{\omega_{p \sigma}^{2}}{c^{2}}\left[\frac{\omega \Omega_{\sigma}}{\Omega_{\sigma}^{2}-\omega^{2}}\left(\frac{\omega^{2}}{\Omega_{\sigma}^{2}}-\frac{2 k_{\perp}^{2} T_{i}}{M_{\sigma} \Omega_{\sigma}^{2}}\right)+\frac{\omega \Omega_{\sigma}}{4 \Omega_{\sigma}^{2}-\omega^{2}}\left(\frac{2 k_{\perp}^{2} T_{i}}{M_{\sigma} \Omega_{\sigma}^{2}}\right)\right] \\
& W_{\sigma}=W\left(\xi_{\varepsilon}\right)=\frac{1}{\sqrt{\pi}} \int_{-\infty}^{\infty} \frac{x e^{-x^{2}}}{x-\xi_{e}-i \varepsilon} d x \\
& \xi_{e}=\frac{\omega}{k_{11}}\left(\frac{m}{2 T_{e}}\right)^{1 / 2} \\
& D_{e}^{2}=T_{e} / 4 \pi n e^{2}
\end{aligned}
$$

Formulas (22-24) are based on the inequalities

$$
\frac{\left(\omega-\Omega_{\sigma}\right)}{k_{11}}\left(\frac{M_{\sigma}}{2 T_{i}}\right)^{1 / 2} \gg 1 \quad \frac{\omega-2 \Omega_{\sigma}}{k_{\prime \prime}}\left(\frac{M_{\sigma}}{2 T_{i}}\right)^{1 / 2} \gg 1
$$

so that ion-cyclotron damping effects are small. When we wish to consider cyclotron damping processes, we will use the replacement

$\frac{1}{n \Omega_{\sigma}-\omega}-\frac{1}{k_{11}}\left(\frac{M_{\sigma}}{2 \pi T_{i}}\right)^{1 / 2} \int_{-\infty}^{\infty} \frac{e^{-x^{2}} d x}{x-\xi_{n}-i \varepsilon} \equiv \frac{1}{k_{11}}\left(\frac{M_{\sigma}}{2 T_{i}}\right)^{1 / 2} z\left(\xi_{n}\right)$

where

$$
\xi_{n}=\frac{\omega-n \Omega_{\sigma}}{k_{11}}\left(\frac{M_{\sigma}}{2 T_{i}}\right)^{1 / 2}
$$

The cognoscenti will also recognize that the 2-3 and 3-2 elements of the conductivity tensor should not be precisely zero unless $\left|\xi_{e}\right| \gg 1$. These 
and other neglected terms contribute to the weak electron damping of otherwise undamped magnetosonic waves. Our use of $\mathrm{w}_{e}$ only in the 3-3 element adequately describes the electron Landau damping of short wavelength converted waves which is the topic of principal interest here.

Setting the determinant of the coefficient equal to zero yields the dispersion relation

$$
\begin{aligned}
& \left\{\left[c-k_{11}^{2}-k_{\perp}^{2} \sum_{\sigma} \frac{\beta_{\sigma}}{2}\left(\omega^{2}-2 \Omega_{\sigma} \omega\right)\left(\frac{1}{\Omega_{\sigma}^{2}-\omega^{2}}-\frac{1}{4 \Omega_{\sigma}^{2}-\omega^{2}}\right)\right]\right. \\
& {\left[B-k_{11}^{2}-k_{\perp}^{2} \sum_{\sigma} \frac{\beta_{\sigma}}{2}\left(\omega^{2}+2 \Omega_{\sigma} \omega\right)\left(\frac{1}{\Omega_{\sigma}^{2}-\omega^{2}}-\frac{1}{4 \Omega_{\sigma}^{2}-\omega^{2}}\right)\right]} \\
& \left.-k_{\perp}^{2}\left(1+\sum_{\sigma} \beta_{\sigma} \frac{\omega^{2}}{\Omega_{\sigma}^{2}-\omega^{2}}\right)\left[A-k_{11}^{2}-k_{\perp}^{2} \sum_{\sigma}^{B_{\sigma}} \frac{\omega^{2}}{2}\left(\frac{\omega^{2}}{\Omega_{\sigma}^{2}-\omega^{2}}-\frac{\omega^{2}}{4 \Omega_{\sigma}^{2}-\omega^{2}}\right)\right]\right\} \\
& {\left[1-\frac{k_{\perp}^{2} c^{2} D_{e}^{2} k_{11}^{2}}{\omega^{2} w_{e}}\right]} \\
& =\frac{k_{\perp}^{2} c^{2} D_{e}^{2} k_{11}^{2}}{\omega^{2} w_{e}} k_{11}^{2}\left[A-k_{11}^{2}-k_{\perp}^{2}-k_{\perp}^{2} \sum_{\sigma} \beta_{\sigma}\left(\frac{3 \omega^{2}}{2\left(\Omega_{\sigma}^{2}-\omega^{2}\right)}-\frac{\omega^{2}}{2\left(4 \Omega_{\sigma}^{2}-\omega^{2}\right)}\right)\right]=0
\end{aligned}
$$

where

$\beta_{\sigma}=\frac{8 \pi \mathrm{n}_{\text {io }} \mathrm{T}_{\mathrm{i}}}{\mathrm{B}^{2}}$

and A, B, C are given by $(5-7)$ and Fig. 1 .

Fortunately, equation (31) permits a considerable simplification. The procedure is to collect powers of $k_{i}^{2}$ and examine the contributions to the coefficients. We shall discard terms that are (1) finite $\beta$ contributions 
with a fundamental resonance denominator $\left(\Omega_{\sigma}^{2}-\omega^{2}\right)^{-1}$ to coefficients where there is already a cold plasma term (i.e., A, B) with a fundamental resonance denominator, (2) contributions to coefficients that are of the order $A \frac{c^{2} k_{\|}^{2} D_{e}^{2}}{\omega^{2} W_{e}} \backsim \frac{T_{e} k_{\prime \prime}^{2}}{M \omega^{2} W_{e}} \ll 1$

relative to other contributions to the coefficient, or (3) of order 3 relative to other contributions. The simplified dispersion relation is then

$$
\begin{aligned}
& k_{\perp}^{6} \frac{c^{2} D_{e}^{2} k_{11}^{2}}{\omega^{2} W_{e}} \sum_{\sigma} \frac{\beta_{\sigma}}{2}\left(\frac{\omega^{2}}{4 \Omega_{\sigma}^{2}-\omega^{2}}-\frac{\omega^{2}}{\Omega_{\sigma}^{2}-\omega^{2}}\right) \\
& +k_{\perp}^{4}\left[A \frac{c^{2} D_{e}^{2} k_{11}^{2}}{\omega^{2} W_{e}}+\sum_{\sigma} \frac{\beta \sigma}{2}\left(\frac{\omega^{2}}{\Omega_{\sigma}^{2}-\omega^{2}}-\frac{\omega^{2}}{4 \Omega_{\sigma}^{2}-\omega^{2}}\right)\right] \\
& -k_{\perp}^{2}\left[\left(A-k_{11}^{2}\right)-\left(C-k_{11}^{2}\right) \underset{\sigma}{[} \beta_{\sigma} \frac{\omega^{2}}{4 \Omega_{\sigma}^{2}-\omega^{2}}\right]+\left(B-k_{11}^{2}\right)\left(C-k_{11}^{2}\right)=0
\end{aligned}
$$

In the slab genmetry. of Fig. 5, one has to have a good approximation

$$
\begin{aligned}
& k_{11}=k_{z}+\frac{\vec{B}_{p} \cdot \vec{k}_{1}}{B}=k_{z}+\varepsilon k_{x}+0\left(\varepsilon k_{y}\right) \\
& \vec{k}_{1}=\vec{x}_{x}+\vec{y} k_{y}
\end{aligned}
$$

where

$\varepsilon \equiv\left(B_{p} / B\right) \cos \theta=(r / R q) \cos \theta \ll 1$

$q$ being the usual tokamak safety factor. 
Snell's law demands that waves propagatc with fixed $\omega, k_{z}, k_{y}$. Only the $\mathrm{x}$-component of the wave number is permitted to vary so that the dispersion relation remains satisfied as the equilibrium quantities, $A, B, \Omega_{\sigma}$, vary.

The mode conversion and absorption phenomena governed by (32) are best brought out by discussions of particular cases.

A. Minority protons in a deuterium plasma. This case corresponds to the present experiments in ATC, TFR, and TM-IHF [1-3]. It has the unique feature that the second harmonic of the major species is degenerate with the fundamental of the minority species and thus close to the ion-hybrid resonance frequency. Fig. 2 informs us that the peripheral region where ion-cyclotron mode conversion takes place is small, and hence we neglect it. Since the mode conversion takes place largely in high density plasma, we can also neglect the $k_{z}$ contributions to $k_{11}$ and, after some manipulation and discarding of small terms, we arrive at the dispersion relation

$$
\begin{aligned}
& s^{8} \frac{\beta_{e} \varepsilon^{2}}{8 W_{c}} \frac{\beta_{D} R}{4 x}+s^{6} \frac{B_{c} \varepsilon^{2}}{8 W_{e}}\left(-\frac{4}{3}+\frac{n_{p}^{R}}{x}\right)+s^{\prime 1}\left(\varepsilon^{2}-\frac{\beta_{D}^{R}}{4 x}\right)-s^{2}\left(-\frac{1}{3}+\frac{n_{p}^{R}}{x}\right) \\
& +\frac{16}{3}\left(-1+\frac{\eta_{p} R}{2 x}\right)=0
\end{aligned}
$$

where $s=k_{x} c / \tilde{\omega}_{p D}, B_{e, D}$ refer to that of the electrons and deuterium ions, and $x$ represents the distance inward in major radius from the $\omega=2 \Omega_{\mathrm{D}}$ surface. The argument of the $w_{e}$ function is

$\frac{\omega}{k_{11}}\left(\frac{m}{2 T_{c}}\right)^{1 / 2}=\frac{2 \Omega_{D} c}{\varepsilon S \omega_{p D}}\left(\frac{m}{2 T_{c}}\right)^{1 / 2}=\frac{2}{\varepsilon S}\left(\frac{m}{M \beta_{e}}\right)^{1 / 2} \leqslant 1$, 
while the Ion damping can be ignored provided

$\frac{\omega-2 \Omega_{\mathrm{D}}}{\varepsilon \mathrm{k}_{\mathrm{x}}}\left(\frac{\mathrm{m}}{2 \mathrm{~T}_{\mathrm{i}}}\right)^{1 / 2}=\frac{2 \mathrm{x}}{\mathrm{RS} \varepsilon \beta_{\mathrm{D}}^{1 / 2}} \gg 1$

Let us also show that the term in $\mathrm{s}^{6}$ is negligible. Treating the three highest powers of $S$ as a quadratic, equation, one finds that the $S^{6}$ term is negligible provided $b^{2} \ll 4 a c$ which yields

$\frac{\beta_{e}^{2} \varepsilon^{4}}{64}\left(\frac{4}{3}-\frac{\eta_{p} R}{x}\right)^{2} \ll \frac{\beta_{e} \varepsilon^{4} \beta_{D}}{8}\left|\frac{R}{x}\left(1-\frac{\beta_{D}^{R}}{4 x \varepsilon^{2}}\right)\right|$

an inequality true since $\mathrm{R}|\mathrm{x}|^{-1} \gg 1$ for tokamaks. Hence the dispersion relation of interest is

$\frac{s^{8} \beta_{e} \beta_{D} R^{2}}{32 W_{e} x}+s^{4}\left(\varepsilon^{2}-\frac{\beta_{D} R}{4 x}\right)+s^{2}\left(\frac{4}{3}-\frac{\eta_{p} R}{x}\right)-\frac{16}{3}\left(1-\frac{\eta_{p} R}{2 x}\right)=0$

What physics can one extract from equation (40)? It is evident that the coefficients of $s^{2}$ and $s^{4}$ can vanish, indicating that two mode conversion processes will take place. Let us follow the wave energy through the mode conversion processes until we can establish that it is being absorbed via a collisionless damping process. By comparing the values of $x$ at which the coefficients of $s^{4}$ and $s^{2}$ vanish, one establishes that the details of the mode conversion process depend upon the value of the parameter $3 \varepsilon^{2} n_{\mathrm{p}} / \beta_{\mathrm{D}}$. We shall examine both limits: (a) $3 \varepsilon^{2} n_{\mathrm{P}} / \beta_{\mathrm{D}} \gg 1$ and (b) $3 \varepsilon^{2} n_{P} / \beta_{D} \ll 1$. Limit (a) applies to most of the region of present day experiments as the nominal values for the ATC experiment demonstrate (see Table 1). Limit (b) applies to the higher $B$ experiments planned for the 
future as well as regions in present experiments where $\varepsilon$ is small because $\mathrm{r} \cos \theta$ is small. Figure 6 sketches the roots of the dispersion relation in the two limits. Both graphs make the same point: Energy can propagate away from the mode conversion region only along the propagating part of the 8-4 branch of the dispersion relation. As this branch of the dispersion relation crosses the cyclotron resonance surface, ion-cyclotron damping by deuterium takes place. Utilizing $(29),(38)$ and $(40)$, one finds that the width of the deuterium cyclotron damping region is

$\delta x_{C} \sim \operatorname{RS} \varepsilon \beta_{D}^{1 / 2} \approx(8)^{1 / 4} R \varepsilon^{1 / 2} \beta_{D}^{1 / 2} \beta_{e}^{-1 / 4}$

while the spatial damping decrement is determined from the equation

$$
s^{4}=\frac{8}{\varepsilon^{2} \beta_{e}}-\frac{32 x}{R \beta_{e} \beta_{D}} \rightarrow \frac{8}{\varepsilon^{2} \beta_{e}}-\frac{16 \cdot k_{1 r} \cdot \bar{v}_{c}}{\Omega_{D} \beta_{e} \beta_{D}} \frac{z^{*}}{|z|^{2}}
$$

with $k_{11}=S \varepsilon \omega_{\mathrm{pD}} \mathrm{c}^{-1}$. Using a perturbation approach one finds that

$\operatorname{Im} S_{D} \approx \frac{4 \varepsilon^{2}}{(8 \pi)^{1 / 2} \beta_{e}^{1 / 2} \beta_{D}^{1 / 2}}$

and that

$\delta x_{c} \frac{\omega_{P D}}{c} \operatorname{Im} S_{D} \approx \frac{\omega_{p D^{R}}}{c} \frac{\varepsilon^{2.5}}{\beta_{e}^{0.75}} \gg 1$

where nominal values have been used.

Inequality (43) assures us that energy converted into the $8-4$ branch of the dispersion relation will be absorbed by deuterium ion-cyclotron damping when it finally reaches the cyclotron resonance layer. On the 
other hand, electron damping operates throughout the mode conversion region. On the 8-4 branch, one can use (40) to obtain

$\mathrm{ImS}_{\mathrm{e}} \approx \frac{1}{2} \sqrt{\pi} \varepsilon^{-1} B_{\mathrm{e}}^{-1 / 2}(\mathrm{~m} / \mathrm{M})^{1 / 2}$

while the characteristic distance comes from the separation of the $(4-2) \rightarrow(8-4)$ mode conversion layer from cyclotron resonance surface: $\delta x_{M C} \approx \beta_{D} R / 4 \varepsilon^{2}$.

Hence the total attenuation due to electrons is

$\exp \left[-\frac{\omega_{p D}}{c}\left(\operatorname{ImS} S_{e} \frac{\beta_{D} R}{4 \varepsilon^{2}}\right] \approx \exp (-5)\right.$

a strong attenuation which appears not to permit propagation of the $8-4$ branch to the cyclotron layer. However, the cyclotron layer and mode conversion point are roughly coincident because

$\frac{\delta x_{c}}{\delta x_{M c}} \approx \frac{4(8)^{1 / 4} \varepsilon^{2.5}}{\beta_{D}^{1 / 2} B_{e}^{1 / 4}} \cdots 1$

pointing out that both cyclotron absorption and electron damping will take place. The ratio of the two attenuation coefficients is

$\frac{\operatorname{ImS} S_{D}}{\operatorname{ImS}}=\frac{\sqrt{8}}{\pi} \frac{\varepsilon^{3}}{\beta_{D}^{1 / 2}}\left(\frac{M}{m}\right)^{1 / 2} \sim \frac{1}{3}$

suggesting that electron absorption is larger than deuterium ion-cyclotron absorption, but not by a large factor. Proton cyclotron effects play no role on the $8-4$ branch. 
In summary, minority protons in a deuterium plasma produce an ionhybrid mode conversion absorption of magnetosonic waves. The converted waves are damped, in roughly equal proportions, by second harmonic deuterion cyclotron absorption and electron Landon damping. In all our work, the parameter $\varepsilon$ plays a key role pointing out the importance of the rotational transform.

B. Deuterium-Tritium Plasmas. The deuterium-tritium plasma provides a good example of ion-hybrid mode-conversion processes and in particular, the case of a minorlty heavy ion in a light ion plasma. In addition, degeneracies between second harmonic and fundamental frequencies are absent. We shall develop our equations for the case where tritium is a minority ion, but our results apply qualitatively up to tritium concentrations of roughly $50 \%$. Figures 3 and 4 point out that no less than three mode conversion processes occur in this case - tritium and deuterium ion-cyclotron mode conversion as well as the ion-ion hybrid mode conversion resonance. Let us concentrate here on the ion-hybrid mode conversion; ion-cyclotron mode conversion processes which occur only at the edge of the plasma will be discussed below.

The appropriate dispersion relation comes from (32) and is

$$
\begin{aligned}
& s^{8} \frac{9 \varepsilon^{2} \beta_{e} \beta_{D}}{32 W_{e}}\left(-\frac{27}{20}+\frac{\eta_{T}^{R}}{x}\right)+s^{6} \frac{3 \varepsilon^{2} \beta_{e}}{8 W_{e}}\left(\frac{12}{5}-\frac{R \eta_{T}}{x}\right) \\
& +s^{4}\left(\varepsilon^{2}+\frac{27 \beta_{e}}{80}-\frac{\beta_{D} \eta_{T} R}{4 x}\right)-\frac{s^{2}}{3}\left(\frac{12}{5}-\frac{R n_{T}}{x}\right)+\frac{8}{45}\left(2-\frac{\eta_{T}^{R}}{x}\right)=0
\end{aligned}
$$

where we have included first and second harmonic deuterium contributions in the coefficients of $s^{4}$ and $s^{8}$. We shall also assume that $\beta_{D} \ll \varepsilon^{2}$, as is generally appropriate for existing and planned tokamaks. But, in the reactor regime, this inequality may not be true. 
Evidently, the coefficients of all powers of $\mathrm{s}^{2}$ can vanish indicating that the mode conversion problem posed by (48) is not well-defined. But the highest powers of $S$ generate values of $s^{2}$ given by $s^{2} \sim \beta^{-1}$ (i.e., $\mathrm{k}_{1}^{2} \mathrm{~T} / \mathrm{M} \Omega^{2} \sim 1$ ) where our finite Larmor radius expansion procedure has broken down. Hence we must try to simplify the physics to get a we11-posed mathematical problem, consistent with our FLR expansion.

Our simplification will consist of ignoring the $s^{8}$ term completely, and dropping the $12 / 5$ contribution to the coefficient of $s^{6}$. The physical basis for this simplification originates with Fig. 7 which shows the mode conversion diagram approrpiate to the simplified dispersion relation

$-s^{6} \frac{3 \varepsilon^{2} \beta_{e}}{8 W_{e}}\left(\frac{R n_{T}}{x}\right)+s^{4} \cdot \varepsilon^{2}-\frac{s^{2}}{3}\left(\frac{12}{5}-\frac{R}{x} \eta_{T}\right)+\frac{8}{45}\left(2-\frac{n_{T} T^{R}}{x}\right)=0$

and contrasts it with that of (48).

The key difference is that in our simplified model the branch of the dispersion relation labeled A permits propagation of energy away from the mode conversion layer, whereas the true one does not. On the other hand, the scale lengths associated with branch $\mathrm{A}$ are always short compared to those of branches $B$ and $C$ which connect to the cold plasma fast-wave branch. Hence the coupling between branch $\mathrm{A}$ and branches $\mathrm{B}$ and $\mathrm{C}$ will be weak and $\mathrm{a}$ simplified. model of branch A will suffice. Our full wave calculation carried out in equations (68) to (75) reaches the same conclusion.

How is the energy absorbed in this mode conversion situation? Figure 7 shows that: the energy absorbed from the fast wave will appear on the short wavelength portion of branch $B$, propagating away from the tritium cyclotron resonance surface. In terms of physics, this wave is a deuterium electro- 
magnetic ion-cyclotron wave, and the principal damping mechanism is the electron damping associated with the $s^{6}$ term of (49). Computing the spatial damping decrements one obtains

$\operatorname{Im} S_{e} \approx \frac{4 \sqrt{\pi}}{15} \frac{\beta_{e}^{1 / 2}}{\varepsilon^{3}}\left(\frac{m}{M}\right)^{1 / 2} \sim 1$

which is sufficient to damp the wave within a distance $c / \omega_{\mathrm{pD}} \sim 10 \mathrm{~cm}$ of the mode conversion layer.

Qualitatively, the prediction is that for future, larger tokamaks, mode conversion at the tritium-deuterium ion hybrid resonance leads to a fairly local electron heating. This prediction holds for any heavy minority ion in a plasma composed of lighter ions. Again, $\varepsilon$ and the rotational transform play crucial roles. One can also readily check that for $\eta_{T}>10^{-2}$, the cyclotron absorption region is sufficiently far from the mode conversion layer so that tritium cyclotron resonance absorption does not occur.

C. Ion-Cyclotron Mode Conversion. In the low density peripheral regions of a tokamak plasma, Figs. 3 and 4 show that an ion-cyclotron resonance mode conversion process takes place which corresponds to the ion-cyclotron mode conversion processes in single-species plasmas $[4,11]$. In configurations where these mode conversion surfaces occupy a fair fraction of the plasma per1meter, the poloidal field is almost parallet to the mode conversion surface and the quantity $\varepsilon$, defined by (35), is verỳ small. Consequently, rotational transform does not couple the $k_{x}$ and $k_{11}$ wave numbers, and the mode conversion process occurs at constant $k_{11}=k_{2}$. In addition, the parallel wavenumber determines the density at which the mode conversion takes place. We shall treat the simplest form of ion-cyciotron mode - that of a single species 
deuterium plasma - as illustrative of the physics of ion-cyclotron mode conversion. As the frequency falls substantially below the gyrofrequency, ioncyclotron mode conversion heating transforms into the Alfven wave heating process discussed by Hasagawa and Chen [12] and by Tataronis [13].

The dispersion relation which governs ion-cyclotron mode conversion in a deuterium plasma can be obtained from (32) with the following simplifications: (1) The mode conversion is brought about principally by the density variation and so one can write

$\frac{\omega_{\mathrm{pD}}^{2} \omega^{2}}{c^{2}\left(\Omega_{\mathrm{D}}^{2}-\omega^{2}\right)}\left(1+\frac{x}{\mathrm{~L}}\right)-k_{\prime \prime}^{2} \equiv \frac{\omega_{\mathrm{pD}}^{2} \omega^{2}}{c^{2}\left(\Omega_{\mathrm{D}}^{2}-\omega^{2}\right)}\left(\frac{x}{\mathrm{~L}}\right)$

which. serves to define the density gradient scale length $L$. The mode conversion takes place at $x=0$. (2) The relation between $k_{11}^{2}$ and $\omega_{p D}^{2}$ at the mode conversion point is used to evaluate $k_{11}^{2}$ in the $k_{\perp}^{4}$ and $k_{\perp}^{6}$ terms of (32). (3) Because the frequency is close to the deuterium gyrofrequency, only the fundamental gyroresource terms are, retained. (4) The term $C$ is negligible compared to $k_{11}^{2}$ in the $10 \mathrm{w}$ density peripheral regions. Straightforward a1gehra then generates the dispersion relation

$$
\begin{aligned}
& -s^{6} \frac{\beta_{e}^{\beta_{i}}}{4 W_{e}}\left(\frac{\omega^{2}}{\Omega_{D}^{2}-\omega^{2}}\right)+s^{4}\left[\frac{{ }_{e}}{2 W_{e}}\left(\frac{\omega^{2}}{\Omega_{D}^{2}-\omega^{2}}\right)+\frac{\beta_{D}}{2}\right] \\
& -s^{2}\left(\frac{x}{L}\right)-s_{11}^{2}\left[\left(\frac{2 x}{L}\right)+1\right]=0 .
\end{aligned}
$$

where the $\beta^{\prime}$ 's refer to the plasma at the mode conversion surface. The argument of the $\mathrm{w}_{\mathrm{e}}$ function is 
$\xi_{e}=\frac{\omega}{k_{11}}\left(\frac{m}{2 T}\right)^{1 / 2}=\left(\frac{\Omega_{D}^{2}-\omega^{2}}{\Omega_{D}^{2}}\right)^{1 / 2}\left(\frac{m}{M_{D} \beta_{e}}\right)^{1 / 2} \sim 1$

indicating electron damping will be important. Indeed, one can readily calculate that

$\frac{\omega_{\mathrm{pD}} \mathrm{L}}{\mathrm{c}} \operatorname{Im} \mathrm{S} \approx\left(\frac{\Omega_{\mathrm{D}}^{2}-\omega^{2}}{2 \omega^{2}}\right)^{1 / 2} \frac{\mathrm{L}}{\mathrm{a}}\left(\frac{\mathrm{x}}{\mathrm{L}}\right)^{1 / 2} \mathrm{I}_{\mathrm{m}} \mathrm{W}_{\mathrm{e}} \gg 1$

with

$\mathrm{a}^{2}=2 \mathrm{~T}_{\mathrm{e}} / \dot{\mathrm{M}}: \Omega_{\mathrm{D}}^{2}$

so that the mode converted wave is absorbed by electron Landau damping close to the mode conversion surface. Hence ion-cyclotron mode conversion processes lead to surface electron heating.

Figure 8 sketches the mode conversion diagram for ion-cyclotron mode conversion. Evidently, the 6-4 branch of the dispersion relation plays a small role, and the $s^{6}$ term in (51) can be safely neglected.

Summary. The most important result of this section has been the identification of the dominant physical processes as manifested in the various simplified dispersion relations (40), (49), and (51). In addition, we have established that the converted short wavelength modes are all quickly attenuated in space, implying that no reflected energy can propagate into a mode conversion layer. These considerations directly impact on the full wave solution of the next section. Lasțy, by considering what processes coniributed to the spatial damping, we were able to determine whether electron or ion heating should result. For example, equạtions (42) - (47) predict that a fair amount of deuteron heating should occur in present experiments

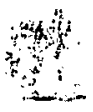


with minority protons. But, in a D-T plasma, equations (49) and (50) predict that ion-ion hybrid mode conversion results in electron heating. The ubiquitous presence of $\varepsilon(e q .35)$ in our formulas indicates the strong influence of the poloidal field on the physics of the converted waves.

\section{FULL WAVE CALCULATIONS OF MODE CONVERSION}

The role of full wave calculations in mode conversion problems is twofold. First, we wish to show that the total energy extracted from the cold plasma branches is the same in the Budden model as in mode conversion models which radiate short wavelength waves away from the mode conversion point. Secondly, full wave calculations are required to show that the short-wavelength energy is in fact radiated inco the expected branches of the dispersion relation. For example, in Fig. $7 \mathrm{~b}$ the energy could in principle be radiated In buth the $A$ and $B$ branches of the dispersion relation. However, because only the $B$ branch goes continuously into a cold plasma branch, we expect most of the radiated energy will appear on that branch. The reader who is interested solely in practical results is advised to read only the summary of this section, leaving the saddle points and asymptotic formulae for the aficionados.

The Budden Model. The Budden model is derived from the ideal model by treating the frequency as a Laplace transform variable with a small positive imaginary part $\omega \rightarrow \omega+i \delta$. Consequently, the coefficlent $A-k_{11}^{2}$ does not precisely vanish, and the coefficients of $k_{\perp}^{2}$ and $k_{\perp}^{0}$ in (32) give rise to the differential equation of the form 
$\frac{\partial^{2} \psi}{\partial \xi^{2}}+\left(1-\frac{\eta}{\xi+i \Delta}\right) \psi=0$

which no longer is singular because of the small positive number $\Delta$. Equation (54) is a confluent hypergeometric equation having a sutoff at $\xi=\eta$ and resonance at $\xi=0$. A similar equation was analyzed by Budden [8]. The precise interpretation of the dependent variable $\psi$ is not yet clear, since (54) has merely been written down by analogy with the dispersion relatinn (32). Derivations of the Budden equation from the differential equation for wave propagation follow in the next section, which will determine the parameter $\eta$ and the nondimensional variable $\xi$.

It should also be pointed out that (54), which yeilds propagating waves for $|\xi| \gg n$, is the appropriate equation for ion-ion hybrid mode conversion in a high density plasma. Ion-cyclotron mòde conversion yields an equation of the form

$\frac{\partial^{2} \psi}{\partial \xi^{2}}-\left(1+\frac{\eta}{\xi+i \Delta}\right) \psi=0$

which produces evanescent waves for $|\xi| \gg n$.

Equation (54) can be solved by Laplace's method [14] with the result

$\psi(\xi)=-\int_{C} \frac{d k}{1-k^{2}} \exp \left[+i k \xi-k \dot{\Delta}=i \eta \int^{k} \frac{d k^{\prime}}{1-k^{\prime 2}}\right]$

and the contour $\mathrm{C}$ must be chosen so that the exponential factor in (56) vanishes or returns to the same valite at the end points. An integration by parts transforms (56) into 
$\psi_{1,2}=\frac{(\xi+i \Delta)}{n} \int_{C_{1,2}}\left(\frac{1+k}{1-k}\right)^{i n / 2} e^{i k \xi-k \Delta} d k$

where Fig. 9 shows two contours. $C_{1}, C_{2}$ corresponding to two independent solutions to (55). The asymptotic formula for these solutions are

$\psi_{1}= \begin{cases}k_{1} \exp (-\pi n / 4) \psi^{+} & \xi>n \\ k_{1} \exp (\pi n / 4) \psi^{+} & \xi \ll-n\end{cases}$

$\psi_{2}= \begin{cases}\mathrm{K}_{2} \exp (\pi n / 4)\left(\psi^{+}+\psi^{-}\right) & \xi \gg n \\ \mathrm{~K}_{2} \exp (-\pi \eta / 4)\left(\psi^{+}+\psi^{-}\right) & \xi \ll-\eta\end{cases}$

where the constants are

$\mathrm{K}_{1}=-\left(e^{\pi n / 2}-e^{-\pi n / 2}\right) n^{-1} ; \quad \mathrm{K}_{2}=-\left(e^{\pi n}-1\right)$

and the functions $\psi^{ \pm}$have the asymptotic formula

$\psi^{+}= \begin{cases}c e^{i \xi} \xi^{-i n / 2} & \xi \gg n \\ c e^{i \xi}|\xi|^{i n / 2} & \xi \ll-n\end{cases}$

$\psi^{-}= \begin{cases}C^{*} e^{-i \xi} \xi^{i n / 2} & \xi \gg n \\ c^{*} e^{-i \xi}|\xi|^{i n / 2} & \xi \ll-n\end{cases}$

and where

$c=i 2^{-i n / 2} \int_{0}^{\infty} l y e^{-y} y^{i n / 2}$. 
These results are the same as Budden's: Solution $\psi_{1}$ corresponds to a wave incident on the resonance with zero reflection coefficient and transmission coefficient $\mathrm{T}=\exp (-\pi n / 2)$. The linear combination

$\psi_{C}=\left(\psi_{2} / K_{2}\right) \exp (\pi n / 4)-\left(\psi_{1} / K_{1}\right) \exp (-\pi n / 4)$

corresponds to a wave incident on the cutoff with reflection coefficient $R=1-e^{-\bar{\pi} \eta}$ and transmission coefficient $T=-\exp (-\pi n / 2)$.

One should also note that $\psi_{2}$ which does not depend on a positive $\Delta$ [as is evident from (56) and contour $\mathrm{C}_{2}$ of $\mathrm{Fig}$. 9] represents the solution of (54) which is regular at the origin. Since this solution involves no singularities, it will not produce any energy absorption - a point made by Swanson [15].

Our principal goal is to demonstrate that the Budden model yields the correct energy absorption. Our arguments that this is so proceed as follows. We shall employ Laplace's method [14] to generate solutions of higher order differential equations corresponding to dispersion relations (40) and (49). We shall then examine what types of contours are allowed in the region $k \sim 1$, and show that any contour which connects to a short wavelength branch propagating away from mode conversion region is of the type $C_{1}$ of Fig. 9 . We shall also establish the contours of type $C_{2}$ are possible. Since the contours $\mathrm{C}_{1}$ and $\mathrm{C}_{2}$ represent independent solutions of the second order differential equation, we conclude that the Budden model satisfactorily describes the energy dissipation. Furthermore, by examining the saddle points which correspond to the short wavelength modes, we shạl demonstrate that energy emerges from the mode conversion region only on short wavelength branches which are 
continuously connected to the ideal branches. This argument justifies our use of W.K.B. concepts in identifying how the mode-converted energy will. eventually heat the plasma.

Let us now provide these results for dispersion relation (40) which governs minority protons in a deuterium plasma. The eighth-order differential equation is:

$$
\left\{\varepsilon_{8} \frac{\partial^{8}}{\partial \xi^{8}}+\dot{\varepsilon}_{4}\left(\xi_{1}-\xi\right) \frac{\partial^{4}}{\partial \xi^{4}}+\xi \frac{\partial^{2}}{\partial \xi^{2}}+(\xi-n)\right\} \psi=0
$$

where

$$
\begin{aligned}
& \varepsilon_{8}=\frac{3 \omega_{\mathrm{pD}}{ }^{R}}{c} \varepsilon^{2} \beta_{\mathrm{e}} \beta_{\mathrm{D}}, \quad \varepsilon_{4}=3 \varepsilon^{2}, \quad \xi=\frac{2 \omega_{\mathrm{pD}} \mathbf{x}}{c} \\
& \xi_{1}=3 n(1-\lambda), \quad \eta=\frac{\eta_{\mathrm{p}} \mathrm{R} \omega_{\mathrm{pD}}}{\mathrm{c}}, \quad \lambda=\beta_{\mathrm{D}} / 3 \varepsilon^{2} \eta_{\mathrm{p}} .
\end{aligned}
$$

The three quantities $\xi_{1}, \eta$, and $\lambda$ are all more or less of order unity while $\varepsilon_{4} \ll 1$ and $\varepsilon_{8} \ll \varepsilon_{4}^{3}$. Laplace's method yields the solution to (61) to be

$\psi=\int_{C} \frac{d k}{1 k^{2} c_{4} k^{4}} \exp \left(i k \xi+i \int^{k} \frac{\varepsilon_{8} k^{\prime 8}+\varepsilon_{4} \xi_{1} k^{\prime 4}-n}{1-k^{\prime 2}=\varepsilon_{4} k^{\prime 4}} d k^{\prime}\right)$

where the exponential term must either vanish or return to the same value at the ends of the contour $C$. Branch. cuts must emanate from the points $k= \pm 1$, $k= \pm i \varepsilon_{4}^{-1 / 2}$ to maintain the $k^{\prime}$-integral single valued. The saddle points of this exponential factor correspond to solutions of the equation

$$
0=-\eta+\xi\left(1-k^{2}\right)+\varepsilon_{4}\left(\xi_{1}-\xi_{3}\right) k^{4}+\varepsilon_{8} k^{8}
$$


which is dispersion relation (40) rewritten. The allowed trajectories of the contour $C$ as $|\hat{k}| \rightarrow \infty$ must satisfy

$\frac{\varepsilon_{8}}{5 \varepsilon_{4}}|k|^{5} e^{5 i \theta} \rightarrow \infty e^{\frac{-i \pi}{2}} e^{2 \pi i n}$

which implies that

$\theta=\frac{-\pi}{10} \pm \frac{2 \pi}{5} \mathbf{n}$

Figure 10 shows that we can find two contours which reproduce the topology of contours $C_{1}, C_{2}$ in the region $k \leqslant 1$, and involve only the saddle point at $\mathrm{k}=\left(\varepsilon_{8} / \Xi_{4}\right)^{1 / 4}$ which corresponds to outgoing energy propagation on the $8-4$ branch of the dispersion relation. [This can be seen by using eighth and fourth order terms of $(40)$ and $x / R=\left(4 \Omega^{2}-\omega^{2}\right) / 8 \Omega_{D}^{2}$.]

Now let us turn to the deuterium-tritium plasma. The sixth order differential equation corresponding to dispersion relation (49) and Fig. $7 \mathrm{~b}$ is

$$
\left\{\varepsilon_{6} \frac{\partial^{6}}{\partial \xi^{6}}+\varepsilon_{4}(\xi-5 \eta) \frac{\partial^{4}}{\partial \xi^{4}}+\xi \frac{\partial^{2}}{\partial \xi^{2}}+\xi-n\right\} \psi=0
$$

where

$\xi=\frac{2}{3} \frac{{ }_{\mathrm{pD}}^{\mathrm{X}}}{\mathrm{c}}, \quad \varepsilon_{6}=\frac{10}{9} \varepsilon^{2} \beta_{\mathrm{c}} \eta, \quad \varepsilon_{4}=\frac{5}{9} \varepsilon^{2}, \quad \eta=\frac{\eta_{\mathrm{T}}{ }^{\mathrm{R}} \omega_{\mathrm{pD}}}{18 \mathrm{c}}$

As a rule, $\varepsilon_{6} \ll \varepsilon_{4}^{2}$. Laplace's metho'd yields the solution tor $\psi$

$\psi=\int_{C} \frac{d k}{1-k^{2}+\varepsilon_{4} k^{4}} \exp \left(i k \xi-i \int^{k} \frac{\eta+\varepsilon_{6} k^{\prime 6}-5 \eta \varepsilon_{4} k^{\prime^{4}}}{1-k^{\prime 2}+\varepsilon_{4} k^{\prime^{4}}} d k^{\prime}\right)$ 
where the exponential term must again have the same value at both ends of the contour $C$. Branch cuts must come from $k= \pm 1, \pm \varepsilon_{4}^{-1 / 2}$ to make the k'-integral single valued. The saddle points associated with exponential factor are given by the equation

$-\varepsilon_{6} k^{6}+\varepsilon_{4} \cdot(\xi+5 n) k^{4}+\xi\left(1-k^{2}\right)-n=0$

which is dispersion relation (49) rewritten. Figure 11 shows the contours and saddle points associated with the integral (70). As $|k| \rightarrow \infty$, the exponential term will vanish at the contour ends provided

$-i \frac{\varepsilon_{6}}{3 \varepsilon_{4}}|k|^{3} e^{3 i \theta} \rightarrow \infty e^{-i \pi+2 \pi i n}$

or

$\theta=-\pi / 6+2 \pi n / 3$

Since one cannot deform the contour and the branch cut of Fig. 11b into the lower half plane until eaddla point $A$ is passed (and still have the saddle point contributions dominate the integral), it is evident that contour $C_{1}$, which corresponds to solution $\psi_{1}$ of (58) of the Budden equation, in principle contains; outgoing radiation in both the $A$ and $B$ branches of the dispersion relation (see Fig. 7b). Contour $C_{2}$, which corresponds to $\psi_{2}$ of (58), again does not couple to the short wavelength branches because it is the solution of the second order equation which is regular at the origin. 'In order to determine the relative importance of the $A$ and $B$ branches, we must evaluate integral (70) along the contour. Because $k^{2} \geq 1 / \varepsilon_{4}>1$ for these branches, the inregral. can be simplified to 
$\psi=k \int_{c} \frac{d k}{k^{2}\left(\varepsilon_{4} k^{2}-1\right)} \exp \left[i k \xi-i \int^{k} \frac{k^{\prime 2}\left(\varepsilon_{6} k^{\prime 2}-5 n\right)}{\varepsilon_{4} k^{\prime 2}-1} d k^{\prime}+\Delta\right]$

where $K$ is a constant determined by the integration near $k \sim 1$ and $\Delta$ is a function which is discontinuous across the cut due to branch cut originating at $\mathrm{k}=1$.

Now we would like to estimate the contributions to $\psi$ coming from saddle points $\mathrm{A}$ and $\dot{\mathrm{B}}$. The integration of the argument of the exponential can be performed with the result that

$$
\begin{aligned}
\frac{W}{\mathrm{~W}}= & \int_{\mathrm{r}^{2}} \frac{\mathrm{dk}}{\left.\mathrm{k}^{2} \mathrm{k}^{2}-1\right)} \exp \left[i k \xi-i \frac{\varepsilon_{6} k^{3}}{\varepsilon_{4}{ }^{3}}+i\left(5 n-\frac{\varepsilon_{6}}{\varepsilon_{/ 1}^{2}}\right) k\right. \\
& \left.+i\left(5 n-\frac{\varepsilon_{6}}{\varepsilon_{4}^{2}}\right) \frac{1}{2} \ln \left[\frac{\varepsilon_{4}^{1 / 2} k-1}{\varepsilon_{4}^{1 / 2} k+1}\right]+\Delta\right]
\end{aligned}
$$

Using the fact that $\varepsilon_{6} / \varepsilon_{4}^{2} \ll 1$ and the substitutions $q=\varepsilon_{4}^{1 / 2} k-1$, $t=q(\xi+5 n) \varepsilon_{4}^{-1 / 2}$ one can rewrite the contributions to (73) coming from the neighborhood of saddle point $B$ as

$$
\left(\frac{\psi}{K}\right)_{B}=\frac{\varepsilon_{4}^{1 / 2}}{2^{1+\frac{i 5 \eta}{2}}} e^{i\left(\frac{\xi+5 n}{\varepsilon_{4}^{1 / 2}}\right)}\left(\frac{\xi+5 \eta}{\varepsilon_{4}^{1 / 2}}\right)^{\frac{-i 5 n}{2}} \int_{C_{B}} \frac{d t e^{i t+\Delta}}{1-\frac{i 5 \eta}{2}}
$$

where $C_{B}$ denotes the contour near saddle point $B$. The contribution from the neighborhood of saddle point A can be calculated to be

$$
\left(\frac{\mu l}{K}\right)_{A}=\varepsilon_{4}^{3 / 4}\left(\frac{\varepsilon_{6}}{\varepsilon_{4}^{2}}\right)^{7 / 4} \frac{e^{\frac{2}{3}\left(\frac{\varepsilon_{4}}{\varepsilon_{6}}\right)^{1 / 2}(\xi+5 \eta)^{3 / 2}}}{(\xi+5 \eta)^{9 / 4}} \int_{C_{A}}^{-i t^{2}+\Delta !} \cdot d t
$$


where $\Delta^{\prime}$ is the discontinuity across the contour associated with the branch. points at $k=1, \varepsilon_{4}^{1 / 2}$. The salient feature in comparing (74) and (75) is to note that the coefficient in (75) is very small compared to that of (74), This is the mathematical manifestation of the physical concept that if two branches of the dispersion relation have dissimilar scales, than there is very little coupling between them. In our case, the scales are different provided $\varepsilon_{6}, \ll \varepsilon_{4}^{2}$. Hence we have shown that most of the mode conversion energ; appears on branch $B$.

Summary. This section has proved the expected results: Solutions of the Budden equation have been shown to correspond with full mode conversion solutions where the short wavelength waves propagate away from the mode conversion surface. Furthermore, a comparison between (74) and (75) showed that the mode converted energy appears on the,branches of the dispersion relation which are directly connected to the cold plasma branches. Hence, one can use Figs. 6-8 to determine where the energy dissipated by a mode conversion resonance appears. And, by calculating the spatial damping decrements on these shortwavelength branches one can decide whether electron or ion heating will occur. This justifies procedure which was followed in equations (42), (44), (47), (49) and (53) of the preceding section.

\section{EFFECT OF RESONANCES ON TOROIDAL EIGEMMODES}

The complicated geometries displayed in Figs. 2-4 make it clear that no simple-yet-precise calculations of the effect of the ion-ion hybrid resonance on toroidal eigenmodes can be made - even with the second order equations of the Budden model. What this section does is to pose simplified geometrical models which will permit semiquantitative conclusions to be drawn regarding 
the effect of the ion-ion hybrid and ion-cyclotron resonances on eigenmodes. Our approach regards the complex parallel wave number as the eigenvaiue to be determined as a function of frequency, density, etc., and utilizes the Budden formulation to obtain the correct dissipation.

Ion-Ion Hybrid Resonance. Our sjmplified model for assessing the effect of the ion-ion hybrid resonance on toroidal eigenmodes is portrayed in Fig. 12. The model assumes: (1) A plasma of unïform density and composition exists between two conducting plates. (2) The magnetic intensity varies in the $x$-direction according to $B=B_{0}(R+x) / R$ so as to create an ion-ion hybrid resonance-cutoff pair. (3) One of the ion species is a minor species so that the ion-ion hybrid resonance frequency is near the gyroresonances for that species. (4) The resonance cutoff pair are closely spaced $\Delta x \ll$ a. (5). The $y$ and $z$ directions are directions of symmetry. Hence we use a Fourier transform in the y-direction and a Laplace transform in the $z$-direction since we are interested in the spatial damping. The z-Lapalce transform produces a Budden model of the resonance region. (6) The electric field $\mathrm{E}_{\mathrm{y}}$ vanishes at the conducting walls. (7) The parallel $E_{z}$ field can be ignòred.

What physics does this model represent? First, in the absence of an ion-- ion hybrid resonance, the model will produce an undamped, propagating eigenmode provided the density is sufficiently high. Secondly, according to Figs. 2-4 the ion-ion hybrid surface occurs in high density regions and this model should adequately describe the energỹ dissipation there. Third, since the resonance cutoff layer is thin, we cain use formulas (57-58) to connect the amplitudes on either side of the layer. Although these formulas are derived for negative imaginary part of $k_{1 i}$ (i.e., a Laplace transform variable), they 
are still valid for modest positive imaginary parts of $k_{11}$ by analytic continuation. Therefore, this model will yield the effect of the ion-ion hybrid resonance on an undamped eigenmode. To within factors of order unity, it does not matter that the undamped eigenmode is oversimplified by ignoring density gradients.

Let us now turn to the task of generating a Budden model equation from the differential equations for wave propagation. As we shall see below, this requires a particular choice of dependent variable, since spatial changes in the wave polarization can cause apparent singularities for an arbitrary choice of dependent variable (e.g. $\left.E_{y}\right)$.

The differential equation for wave propagation

$\vec{\nabla} \times(\vec{\nabla} \times \vec{E})=\frac{4 \omega i \overleftrightarrow{\sigma} \cdot \vec{E}}{c^{2}}$

becomes in our model

$$
\begin{aligned}
& \left(A(x)-k^{2}-k_{11}^{2}\right) E_{x}+i\left(D(x)-k \frac{\partial}{\partial x}\right) E_{y}=0 \\
& -i\left(D(x)+k \frac{\partial}{\partial x}\right) E_{x}+\left(A(x)-k_{11}^{2}+\frac{\partial^{2}}{\partial x^{2}}\right) E_{y}=0
\end{aligned}
$$

where we have assumed that all quantities vary as $\exp \left[i\left(k y+k_{, i} z-\omega t\right)\right]$ and $A, D$ are given by $(3,4)$. Utilizing (4) - (7), let us define $\bar{A}$ and $\bar{C}$ by

$$
\bar{A}=A-k_{11}^{2}, \quad \bar{C}=C-k_{11}^{2}, \quad D-\bar{A}-\bar{C} .
$$

While both $\ddot{A}$ and $D$ vary rapidly near a resonance, $\bar{C}$ does not. In the lláripulations below, $\vec{C}$ will be treated as a constant, while variations in $\bar{A}$ and $D$ will be permitted. 
The procedure for deriving a Budden equation is as follows: Solve (76) for $\mathrm{E}_{\mathrm{x}}$ and substitute into (77) obtaining the second order equation

$\frac{\partial}{\partial x} \frac{1}{\bar{A}-k^{2}}\left(\bar{A} \frac{\partial}{\partial x} E_{y}-k D E_{y}\right)+\frac{D k}{\bar{A}-k^{2}} \frac{\partial E_{y}}{\partial x}+\frac{\bar{A}-D^{2}-k^{2} \bar{A}}{\bar{A}-k^{2}} E_{y}=0$

By introducing the variables

$\Phi_{1}=E_{y}, \quad \phi_{?}=\frac{k}{\bar{C}\left(\bar{A}-k^{3}\right)}\left(\bar{A} \frac{\partial}{\partial x} E_{y}-k E_{y}\right)$

one can recast (78) in terms of two coupled first-order equations

$\dot{\bar{A}} \frac{\partial \phi_{1}}{\partial x}=(\bar{A}-\bar{C}) k \phi_{1}+\frac{\left(\bar{A}-k^{2}\right) \bar{C}}{k} \phi_{2}$

$\overline{\mathrm{A}} \frac{\partial \phi_{2}}{\partial \mathrm{x}}=-(\overline{\mathrm{A}}-\overline{\mathrm{C}}) \mathrm{k} \phi_{2}-\mathrm{k}(2 \overline{\mathrm{A}}-\overline{\mathrm{C}}) \phi_{1}$

Next, one can define the variables

$\phi^{+}=\frac{1}{2}\left(\phi_{2}+\phi_{1}\right) \quad \phi^{-}=\frac{1}{2}\left(\phi_{2}-\phi_{1}\right)$

and obtain from (80) a coupled set of first order equations for $\phi^{+}$and $\phi^{-}$.

$2 \mathrm{k} \frac{\partial \phi^{+}}{\partial \mathrm{x}}=\overline{\mathrm{C}} \phi^{-}+\left(\overline{\mathrm{C}}-2 \mathrm{k}^{2}\right) \phi^{+}$

$2 \overline{\mathrm{A}} \frac{\partial \phi^{-}}{\partial \mathrm{x}}=\left(2 \mathrm{k} \overline{\mathrm{A}}-\frac{\overline{\mathrm{A}} \overline{\mathrm{C}}}{\mathrm{k}}\right) \phi^{-}+\left(4 \mathrm{k} \overline{\mathrm{C}}-4 \mathrm{k} \overline{\mathrm{A}}-\frac{\overline{\mathrm{A}} \overline{\mathrm{C}}}{\mathrm{k}}\right) \phi^{+}$

Straightforward manipulations then lead to an equation of the Budden form for $\phi^{+}$

$\frac{\partial^{2} \phi^{+}}{\partial x^{2}}=\left(k^{2}-2 \bar{C}+\frac{\bar{C}^{2}}{\bar{A}}\right) \phi^{+}$ 
as $\bar{A}$ is the quantity which vanishes (except for the small positive imaginary part). Retracing our steps, one obtains the relations between $\phi^{+}, \phi^{-}$and $E_{y}$

$2 \phi^{+}=\frac{\overline{\mathrm{A}}\left(\dot{\overline{\mathrm{C}}}-\mathrm{k}^{2}\right)}{\overline{\mathrm{C}}\left(\overline{\mathrm{A}}-\mathrm{k}^{2}\right)} \mathrm{E}_{\mathrm{y}}+\frac{\mathrm{k} \overline{\mathrm{A}}}{\overline{\mathrm{C}}\left(\overline{\mathrm{A}}-\mathrm{k}^{2}\right)} \frac{\partial}{\partial \mathrm{x}} \mathrm{E}_{\mathrm{y}}$

$2 \phi^{-}=\frac{2 k^{2} \bar{C}-\bar{A} \bar{C}-k^{2} \bar{A}}{\bar{C}\left(\bar{A}-k^{2}\right)} E_{y}+\frac{k \bar{A}}{\bar{C}\left(\bar{A}-k^{2}\right)} \frac{\partial}{\partial x} E_{y}$

At the conducting boundary, the condition $\mathrm{E}_{\mathrm{y}}=0$ leads to $\phi^{+}=\phi^{-}$, and via (81) to

$k \frac{\partial \phi^{+}}{\partial x}=\left(\bar{C}-k^{2}\right) \phi^{+}$

which is the boundary condition we will use in our eigenvalue calculations. Let us note here that the derivation leading to (82) is also valid for the ion-cyclotron resonance because in the low density peripheral plasmas $\bar{c} \approx-k_{\prime \prime}^{2}$ and hence $\overline{\mathrm{C}}$ is constant. In comparing (78) and (82), one notes that the apparent singularity at $\bar{A}=k^{2}$ has been eliminated. The role of this singulari.ty is simply to make the wave polarization such that

$k \frac{\partial E}{\partial x}+\left(\bar{C}-k^{2}\right) E y=0$

at thi.s point.

Let us next rewrite (82) in terms of actual physical variables. The plasma consists of two species: deuterium and a minor species denoted by the subscript "2". The ion-ion hybrid frequency will then be very close to $\Omega_{2}$ and we can write the formulas for $\bar{A}$ and $\bar{C}$ as 


$$
\begin{aligned}
& \bar{A}=\frac{\omega_{p D}^{2}}{c^{2}}\left(\frac{\Omega_{2}^{2}}{\Omega_{D}^{2}-\Omega_{2}^{2}}-\frac{\Gamma_{2} R}{2 x}-\frac{1}{N}\right) \\
& \bar{C}=\frac{\omega_{p D}^{2}}{c^{2}}\left(\frac{\Omega_{2}^{2}}{\left(\Omega_{D}+\Omega_{2}\right) \Omega_{D}}-\frac{1}{N}\right)
\end{aligned}
$$

where we have assumed that

$$
\Gamma_{2}=2 n_{2} z_{2} / A_{2} \ll 1 \quad x / R=\left(\omega-\Omega_{2}\right) / \Omega_{2} \ll 1,
$$

and used the definition $N \equiv c^{2} k_{11}^{2} / \omega_{p D}^{2}$ introduced following (13). Since our. principal interest lies in the high density regime, we will assume $N \gg 1$ and retain only terms of first order in $\mathrm{N}^{-1}$. Substituting (85) into (82), one obtains the equation

$$
\frac{c^{2}}{\omega_{P D}^{2}} \frac{\partial^{2}}{\partial x^{2}} \phi^{+}=\left\{\bar{k}^{2}-2\left[\frac{\Omega_{2}^{2}}{\left(\Omega_{D}+\Omega_{2}\right) \Omega_{D}}-\frac{1}{N}\right]+\frac{x\left(\frac{\Omega_{2}^{2}}{\left(\Omega_{D}+\Omega_{2}\right) \Omega_{D}}-\frac{1}{N}\right)}{\left(\frac{\Omega_{2}^{2}}{\Omega_{D}^{2}-\Omega_{2}^{2}}-\frac{1}{N}\right) x-\frac{\Gamma_{2} R}{2}}\right\}^{+}
$$

with $\bar{k}^{2}=k^{2} c^{2} / \omega_{p D}^{2}$. If one next introduces $x^{\prime}=x-x_{D}$, where

$x_{D}=\frac{\Gamma_{2}^{R}}{z}\left(\frac{\Omega_{2}^{2}}{\Omega_{D}^{2}-s \Omega_{2}^{2}}-\frac{1}{N}\right)^{-1}$

and performs straightforward algebra in which terms of order $\mathrm{N}^{-2}, \Gamma_{2} \mathrm{~N}^{-1}$ are neglected, the resulting differential equation is

$$
\frac{c^{2}}{\omega_{p D}^{2}} \frac{d^{2}}{d x^{\prime 2}} \phi^{+}=\left[\bar{k}^{2}-\frac{\Omega_{2}^{2}}{\Omega_{D}^{2}}+\frac{1}{N}\left(\frac{\Omega_{D}^{2}+\Omega_{2}^{2} c}{\Omega_{D}^{2}}\right)+\frac{\Gamma_{2} R}{2\left(x^{\prime}+i \Delta\right)}\left(\frac{\Omega_{D}-\Omega_{2}}{\Omega_{D}}\right)^{2}\right] \phi^{+}
$$


where it can be readily verified that a small negative imaginary part of $k_{11}$, leads to a small positive imaginary part of $\overline{\mathrm{A}}$, which is represented by $\Delta$. Evidently, equation (89) has the form of a Budden equation, and the simple rescaling of variables leads to

$\frac{d^{2}}{d \xi^{2}} \phi^{+}+\left(1-\frac{n}{\xi+i \Delta}\right) \phi^{+}=0$

where $\Delta$ has been rescaled,

$\xi=\frac{x^{\prime} \omega_{\mathrm{pD}}}{\mathrm{c}}\left[\frac{\Omega_{2}^{2}}{\Omega_{\mathrm{D}}^{2}}-\overline{\mathrm{k}}^{2}-\frac{1}{\mathrm{~N}}\left(\frac{\Omega_{2}^{2}+\Omega_{\mathrm{D}}^{2}}{\Omega_{\mathrm{D}}^{2}}\right)\right]^{1 / 2}$
$\eta=\frac{\Gamma_{2} R \omega_{\mathrm{DD}}}{2 \mathrm{c}}\left[\frac{\Omega_{2}^{2}}{\Omega_{\mathrm{D}}^{2}}-\overline{\mathrm{k}}^{2}-\frac{1}{\mathrm{~N}}\left(\frac{\Omega_{2}^{2}+\Omega_{\mathrm{D}}^{2}}{\Omega_{\mathrm{D}}^{2}}\right)\right]^{-1 / 2}\left(\frac{\Omega_{\mathrm{D}}-\Omega_{2}}{\Omega_{\mathrm{D}}}\right)^{2}$,

and where boundary condition (84) takes the form

$\overline{\mathrm{k}} \frac{\partial \phi^{+}}{\partial \xi_{j}}=\left[\frac{\left(\frac{\Omega_{2}^{2}}{\left(s_{2}+\bar{s}_{D}\right) \Omega_{D}}-\overline{\mathrm{k}}^{2}\right)}{\left(\frac{\Omega_{2}^{2}}{\Omega_{D}^{2}}-\overline{\mathrm{k}}^{2}\right)^{1 / 2}}+0\left(\frac{1}{\mathrm{~N}}\right)\right] \phi^{+}$

when $\bar{k}, N^{-1} \rightarrow 0$ and the appropriate values are used, formulas (9.1) give the Eame expressions for $n$ and $\xi$, us did the W.K.R. analysis for $p-D$ plasmas $(62,63)$ and $\mathrm{n}-\mathrm{T}$ plasmas $(69)$.

The general solution to (90) takes the form

$\phi^{+} \equiv \psi=\psi_{1}+\mathrm{a} \psi_{2}$

and has, from (58)-(59), the asymptotic formula 
v $\begin{cases}\left(e^{\frac{-\pi \eta}{4}}+a e^{\frac{\pi n}{4}}\right) e^{i \xi} \xi^{\frac{-i \eta}{2}}+a e^{\frac{\pi n}{4}} L e^{-i \xi} \xi^{\frac{i \eta}{2}} & . \xi \gg n \\ \left(e^{\frac{\pi n}{4}}+a e^{\frac{-\pi \eta}{4}}\right) e^{i \xi}|\xi|^{\frac{-i \eta}{2}}+a e^{\frac{-\pi \eta}{4}} L e^{-i \xi}|\xi|^{\frac{+i \eta}{2}} & \xi \ll-n\end{cases}$

where $\mathrm{L}=\mathrm{L}(\eta) \stackrel{\vdots}{=} \mathrm{C} * / \mathrm{C}$ is a phase factor. When $\mathrm{k}_{\|}$has a positive imaginary part, the boundary conditions are applied at

$$
\begin{aligned}
& \xi_{2}=\alpha x_{2}(1-i \varepsilon) \\
& \xi_{1}=-\alpha x_{1}(1-i \varepsilon)
\end{aligned}
$$

where.

$\alpha=\frac{\omega_{\mathrm{PD}}}{\mathrm{c}}\left[\frac{\Omega_{2}^{2}}{\Omega_{\mathrm{D}}^{2}}-\overline{\mathrm{k}}^{2}-\frac{1}{\mathrm{~N}} \frac{\Omega_{2}^{2}+\Omega_{\mathrm{D}}^{2}}{\Omega_{\mathrm{D}}^{2}}\right]^{1 / 2}$

and

$\alpha \varepsilon=\frac{k_{n}\left(\operatorname{Im}_{11}\right)\left(\Omega_{2}^{2}+\Omega_{D}^{2}\right)}{\alpha \Omega_{D}^{2}}$

Application of the boundary condition (92) leads to two formulas for a one based on the condition at $\xi_{2}$, the other on the condition at $\xi_{1}$. Equating these two expressions for a generates the eigenvalue equation

$$
\begin{aligned}
1 & +\exp \left[-2 \alpha x_{2} \varepsilon+i\left(\phi_{2}-2 \alpha x_{2}\right)\right] \\
& =e^{-\pi n}\left\{1+\exp \left[2 \alpha x_{1} \varepsilon+i\left(2 \alpha x_{1}+\phi_{1}\right)\right]\right\}
\end{aligned}
$$

where the phase factors are defined by 


$$
\begin{aligned}
& e^{i \phi_{2}} \equiv L(1+i \beta)\left(\alpha x_{2}\right)^{i n} /(1-i \beta) \\
& e^{i \phi_{1}} \equiv L(1+i \beta)\left|\alpha x_{1}\right|^{i n} /(1-i \beta)
\end{aligned}
$$

and $\beta$ comes from the boundary condition (92)

$$
B \equiv \bar{k}\left(\frac{\Omega_{2}^{2}}{\Omega_{D}^{2}}-\bar{k}^{2}\right)^{1 / 2}\left(\frac{\Omega_{2}^{2}}{\left(\Omega_{2}+\Omega_{D}\right)_{D}}-\bar{k}^{2}\right)^{-1}
$$

While the phases $\phi_{1}, \phi_{2}$ do vary with $n$ and $x$, as a rule their magnitude is smaller and their variation less rapid than the phases $2 \alpha x_{1}$ and $2 \alpha x_{2}$. Consequently $\phi_{1}$ and $\phi_{2}$ are effectively constant with respect to variations in $2 \alpha x_{1}, 2 \alpha x_{2}$.

A separation of (98) into real and imaginary parts yields the two equations

$$
\begin{aligned}
& 1+e^{-2 \alpha x_{2} \varepsilon} \cos \left(2 \alpha x_{2}-\phi_{2}\right)=e^{-\pi \eta}\left[1+e^{2 \alpha x_{1} \varepsilon} \cos \left(2 \alpha x_{1}+\phi_{1}\right)\right] \\
& -e^{-2 \alpha x_{2} \varepsilon} \sin \left(2 \alpha x_{2}-\phi_{2}\right)=e^{-\pi \eta\left[e^{2 \alpha x_{1} \varepsilon} \sin \left(2 \alpha x_{1}+\phi_{1}\right)\right]}
\end{aligned}
$$

for which we have to solve for the two free parameters $\alpha, \varepsilon$. Simple expressions can be obtained for the limits $\pi \eta \gg 1$ and $\pi n \ll 1$. The results are for $\pi \eta \gg 1$

$2 \alpha x_{2}-\phi_{2}=(2 \mathrm{n}+1) \pi$

$\left.2 \alpha x_{2} \varepsilon=e^{-\pi \eta} \mid 1+\cos \left(2 \alpha x_{1}+\phi_{1}\right)\right]$ 
and for $\operatorname{lin} \ll 1$,

$$
2 \alpha\left(x_{1}+x_{2}\right)+\phi_{1}-\phi_{2}=2 n \pi-\pi n \sin \left(2 \alpha x_{1}+\phi_{1}\right)
$$

$2 \alpha \varepsilon\left(x_{1}+x_{2}\right)=\pi \eta\left[1+\cos \left(2 \alpha x_{1}+\phi_{1}\right)\right]$

Evidently, the spatial damping can yanish if $2 \alpha x_{1}+\phi_{1}=(2 n+1) \pi$. This corresponds to the case where the eigenmode is a pure $\psi_{2}$ solution [c.f. (58)] and has no dissipation. In general, the freedom permitted to adjust $\alpha$ by varying $N=k_{11}^{2} c^{2} / \omega_{p D}^{2}$ is required to satisfy conditions (103a) or (104a). With $\alpha$ so determined, the spatial damping does not generally vanish.

Practically, the most interesting case is when $\pi n \ll 1$. Utilizing (91), (96) and (97), we can compute the amount of spatial damping a mode experiences once around the torus to be

$\left(I m k_{1 \prime}\right) 2 \pi R \approx \frac{\pi^{2}}{2 n}\left(\frac{R \omega_{p D}}{c}\right)^{2} \frac{R}{a}\left(\frac{2 Z_{2}}{A_{2}} n_{2}\right) \frac{\left(\Omega_{D}-\Omega_{2}\right)^{2}}{\Omega_{D}^{2}+\Omega_{2}^{2}}$

where $\mathrm{n}$ is the toroidal mode number $\mathrm{k}_{11} \equiv \mathrm{n} / \mathrm{R}$. Unless $n_{2} \leq 10^{-2}$, this damping is severe and toroidal eigenmodes do not exist.

Although the case where $\pi n \gg 1$ appears to have small. damping, the simplifications of the model do not take into account the variable width of the resonance-cutoff points according to Figs. 2-4. Hence if $\pi n \gg 1$, near the center of a machine, there will be regions close to the magnetosonic cutoff (surface $\dot{C}$ in Figs. 2-4) where $\pi n$ will be of order unity, and which will provide the damping of an eigenmode.

Hence, we conclude from (105) that the ion-ion hybrid resonance suppresses toroidal eigenmodes. 
The Single Species Ion-Cyclotron Resonance. Any attempt to heat plasma ions via the fundamental cyclotron resonance must, as Fig. 13 makes clear, involve an ion-cyclotron mode conversion surface. As a rule, this surface is constrained to lie in the low density peripheral plasma, so the absorption resulting from it is weak. On the other hand, the damping of a magnetosonic toroidal eigenmode by fundamentally resonant ions is also weak [4], and it is appropriate to compare the two absorption rates. If one tries to increase the resonant ion absorption by making the resonant ion a minority species in the plasma:[16], then ion-ion hybrid resonance processes come into play as Figs. 2 and 3 demonstrate. Formula (105) then indicates that the dominant absorption is via the ion-ion hybrid resonance.

Figure 14 portrays our slab model for estimating the effects of ioncyclotron mode conversion. The ion-cyclotron mode conversion results entirely from the density variation; the magnetic field is in the $z$-direction and of uniform strength, so that $\Omega \gg \Omega-\omega>0$ everywhere. The scale length $L$ for the plasma density variation liear the edge is taken small so that

$\mathrm{kL} \ll 1, \quad \mathrm{k}_{11} \mathrm{~L} \ll 1$

Consequently, evanescence of the wave amplitude in the density gradient region can be ignored. We shall also estimate that $k a \sim k_{11} a \sim 1$, as is typical of :tokamak applications. But we shall be concerned with large, dense tokamaks so that $\omega_{\mathrm{pD}^{a}}{ }^{a / c} \gg 1$, which is characteristic of most of the planned tokamak heating experiments. The boundary conditions are that $E_{y}=0$ at $x=0$ and that $\partial E_{y} / \partial x=0$ at $x=a$ (which corresponds to a solution which is even around the point $x=a$ ). 
It is evident from Fig. 14 that the mode conversion process operates strictly at the periphery of the plasma. Mathematically, its effect on eigenmodes will be to change the boundary condition at $\mathrm{x}=0$ from the nondissipative condition $E_{y}=0$ to one involving dissipation. Hence our calculation is a two-step one. First, we investigate the small region where the mode conversion takes place to find the new boundary condition. Then, an eigenmode calculation is done for a uniform plasma with the temporal damping determined by the new dissipative boundary condition. Finally, we compare the damping derrement resulting from mode conversion with that caused by fundamental resonancep absorption by ions.

The : equation which governs mode conversion is (82). Let us simplify notation by renaming the dependent variable $\phi^{+} \rightarrow \psi$, and noting that when $\overline{\mathrm{A}} \approx 0$, $\bar{C}$ is accurately given by $\bar{C}=-k_{11}^{2}$ provided $(\Omega-\omega) / \Omega \ll 1$. Hence, the equation appropriate to the mode conversion region is

$$
\frac{\partial^{2} \psi}{\partial x^{2}}=\left\{k^{2}+k_{11}^{2}\left[\frac{\frac{2 \omega_{p D}^{2} \times \omega^{2}}{c^{2} L\left(\Omega^{2}-\omega^{2}\right)}-k_{11}^{2}}{\frac{\omega_{p D}^{2} \times \omega^{2}}{c^{2} L\left(\Omega^{2}-\omega^{2}\right)}-k_{11}^{2}}\right]\right\} \psi
$$

Introducing the non-dimensional variable $=\mathrm{x} / \mathrm{x}_{\mathrm{D}}$, one transforms (107) into

$\frac{\partial^{2}}{\partial \xi_{3}^{2}} \psi=\left\{\mathrm{k}^{2} \mathrm{x}_{\mathrm{D}}^{2}+\mathrm{k}_{11}^{2} \mathrm{x}_{\mathrm{D}}^{2}\left[\frac{2 \xi_{z}-1}{\xi-1+i \Delta}\right]\right\}$

where

$$
x_{D}=L \cdot\left[\left(\Omega^{2}-\omega^{2}\right) / \omega^{2}\right]\left(k_{11}^{2} c^{2} / \omega_{p D}^{2}\right)
$$


and the small imaginary part $\Delta$ is associated with a Laplace-transform interpretation of $\omega$. The inequalities discussed above serve to establish that $k_{11}^{2} x_{D}^{2} \ll 1$ and $k^{2} x_{D}^{2} \ll 1$. Mathematically, the principal effect of (108) is to provide a discontinuity in slope as the $\xi=1$ point is passed.

$\int \frac{\partial^{2} \psi}{\partial \xi^{2}} \mathrm{~d} \xi=\left(\frac{\partial \psi}{\partial \xi_{3}}\right)_{1}+^{-}\left(\frac{\partial \psi}{\partial \xi}\right)_{1}=-1 \pi k_{11}^{2} k_{D}^{2} \psi$

Combining (110) with (84), we find that the houndary condition for the eigenmode calculation at $\mathrm{x}=0$ is.

$$
\frac{1}{\psi} \frac{\partial \psi}{\partial x}=-\frac{k_{11}^{2}+k^{2}}{k}-i \pi k_{11}^{2} x_{D}
$$

Combining (83) and (81) with inequalities $\bar{A} \gg \bar{C} \gg k^{2}$ appropriate to the dense plasma, and using the condition $\partial \mathrm{E}_{\mathrm{y}} / \partial \mathrm{x}=0$, one finds that the appropriate boundary condition at $\mathrm{x}=\mathrm{a}$ is

$$
\frac{1}{\psi} \frac{\partial \psi}{\partial x}=-2 k
$$

In the high density plasma, the equation governing the eigenmode is

$$
\frac{\partial^{2} \psi}{\partial x^{2}}=\left[k^{2}+2 k_{11}^{2}-\frac{\omega_{p D o}^{2}}{c^{2}}\left(1-\frac{3}{2} \frac{i \gamma}{\Omega}\right)\right] \psi
$$

where we used $\omega \approx \Omega$ and assumed $\gamma>0$ represents a temporal damping of the wave. The Lwo solutions to (113) are evidently

$$
\psi_{1}=\exp (i \alpha x+\delta x) ; \quad \psi_{2}=\exp (-i \alpha x-\delta x)
$$


where

$\alpha=\left(\omega_{\mathrm{pD}}^{2} c^{-2}-k^{2}-k_{11}^{2}\right)^{1 / 2} ; \quad \delta=\frac{3 \gamma \omega_{\mathrm{pD}}^{2}}{4 \mathrm{c}^{2} \alpha \Omega}$

Writing the general solution as $\psi=\psi_{1}+C_{2} \psi_{2}$, and applying boundary conditions (111) and (112) leads to the complex eigenvalue equation

$$
\left(\frac{i \alpha+\delta+\left(k_{11}^{2}+k^{2}\right) k^{-1}+i \pi k_{11}^{2} x_{D}}{i \alpha+\delta-\left(k_{11}^{2}+k^{2}\right) k^{-1}-i \pi k_{11}^{2} x_{D}}\right)=\left(\frac{i \alpha+\delta+2 k}{i \alpha+\delta-2 k}\right) e^{2 i \alpha a+2 \delta a}
$$

The solution to $(116)$ is obtained by iteration. Assume first that $\delta$ and $\pi k_{11}^{2} x_{D}$ can be ignored. We then find that $e^{2 i \alpha_{a}}=e^{i \phi}$, with $\phi \approx 2\left(\mathrm{k}^{2}-\mathrm{k}_{11}^{2}\right) \mathrm{c}^{2} / \omega_{\mathrm{pD}}^{2} \ll 1$, so that $\alpha$ is determined b.y

$\alpha a \approx n \pi$

Also, $\delta$ is given by

$\delta=\frac{\pi k_{1 i}^{2} x_{D}}{a \alpha}$

where terms of relative contribution $\mathrm{k} / \mathrm{a} \alpha^{2} \sim . \mathrm{k}^{2} / \alpha^{2} \ll 1$ have been neglected. Combining (109), (115), and (118), one finds the damping decrement $\gamma$ to be

$\frac{\gamma}{\Omega}=\frac{4 \pi}{3}\left(\frac{k_{11}^{2} c^{2}}{\omega_{p D o}^{2}}\right)^{2} \frac{L}{a}\left(\frac{\Omega^{2}-\omega^{2}}{\omega^{2}}\right)$

For tokamak applications we can estimate that $\left(\Omega^{2}-\omega^{2}\right) \omega^{-2} \approx 2 \mathrm{a} / \mathrm{R}$ and

$L \approx a / 3$ to obtain

$\frac{\gamma}{\lambda}=\pi\left(\frac{k_{11}^{2} c^{2}}{\omega_{p D o}^{2}}\right)^{2}: \frac{a}{R}$. 
which is to be compared to the resonant ion damping decrement [4] of

$$
\frac{\gamma}{\Omega} \approx \frac{\beta_{i}}{2}\left(\frac{k_{11}^{2} c^{2}}{\omega_{p D o}^{2}}\right) \frac{R}{a} .
$$

For planned tokamak experiments, the mode coupling damping decrement . dominates for $\beta_{i}<10^{-2}$, indicating that fundamental resonance heating of tokamak plasmas is noc eflicicnt. Most nf the energy will go into the edge electron heating associated with the ion-cyclotron mode conversion - a conclusion that agrees with the results of the ST-Tokamak heating experiment [17]. A qualitative prediction of $(120)$ is that modes with the smallest values of $k_{11}$ will have the highest $Q$ toroidal eigenmodes.

Our treatment of the ion-cyclotron mnde-conversion process differs from the one implicit in the work of. Hosea and Sinclair [18]. They used a strictly cold plasma theory to describe the short wavelength waves radiated by the mode conversion layer. Since no damping processes were available to these waves in the cold plasma model, they reflected from the wall and returned to the mode conversion layer and hence did not give rise to a net dissipation. . The tulal systcm, ion-cyrintron plus short wavelength waves, propagated undamped. By constrast, the short wavelength waves according to our hot plasma theory are rapidly attenuated by electron Landau damping [c.f. (53)] and hence produce a net damping of an eigenmode. 


\section{DISCUSSION AND TOKAMAK APPLICATIONS}

Let us begin this section with a, summary of our principal arguments and results, hoping that the reader will thus see the forest instead of the trees, branch outs, and poles.

It was shown following (10) that the ideal, cold plasma equations which govern propagation of the fast magnetosonic and electromagnetic ion-cyclotron modes break down along certain surfaces; which are called lun-lon hybrid resonance or ion-cyclotron resonance surfaces. The geometry of these surfaces is sliuwn by curves " $A$ " in Figs. 2-4, where it is seen that the ion-cyclotron and ion-ion hybrid resonance surfaces are actually. continuations of one another, the term ion-cyclotron resonance applying to the low density. regions and vice-versa. There are three principal points made by Figs. 2-4: First, the ion-ion hybrid resonance surface can occur in the high density central regions of a tokamak where it affects. the propagation of the fast magnetosonic mode. Indeed, if the applied frequency is close to the cyclotron frequency of a minority ion, then ion-inn hybrid resonance effects are impossible to avoid, as shown by Figs. 2 and 3. Second, ion-cyclotron resonance surfaces are impussible to avoid if the frequency is close to the cyclotron frequency of a plasma ion, as is the case in proposed fundamental resonance heating experiments (see also Fig. 13). Third, the geometry of the resonance surfaces is complicated, precluding simple-yet-precise models of the energy absorption caused by these surfaces. Instead, we use simple one-dimensional idealizations (Fig. 5) based on the approximation that the resonance layers are thin. These calculations provide semi-quantitative estimates of the effects of resonance layers. The accuracy of the final formulas in this paper is roughly a factor 
of three. But they do provide the appropriate scaling laws and estimates of being large or small.

What physical processes occur at the resonant surfaces? When hybrid order finite Larmor radius, finite conductivity, poloidal field, and hot plasmas effects are added to the wave propagation equation, as discussed in the several paragraphs preceding (17), the wave equation no longer breaks down but has seveid branches nf the dispersion relation. The result is that energy leaves the "ideal" branch of the dispersion relation and, via mode conversion [19], emerges on the short wavelength branches, where it is absorbed by various collisionless absorption processes. The poloidal field parameter . $\varepsilon$, defined in (35), plays an important role in the propagation and absorption of the short wavelength waves generated by an ion-ion hybrid layer. But it is argued, in the paragraphs preceding (51), that the poloidal field is not important in ion-cyclotron mode conversion.

Several specific cases of the general dispersion relation (32) are discussed: The case of minority protops in a deuterium plasma corresponds to present experiments [1-3], but has the unique feature not found in fusion DT plasmas, that the second harmonis cyclotron frequency of the majority species is degenerate with the fundamental cyclotron frequency, of the minority species which is very close to the ion-ion hybrid resonance frequency. The appropriate dispersion relation is (40) whose roots are sketched in Fig. 6 . Equations (42-47) show that energy emerging on the 8-4 branch of the dispersion relation is absorbed by electron Landon damping and deuteron second harmonic cyclotron damping, with the electron damping being roughly a factor of three larger. This agrees with the experimental results [1] that show a modest but not high efficiency of ion heating. 
For the case of a dcuteriun-tritium plasma, dispersion relations (48-49), Fig. 7, and equation (50) show that all the energy absorbed by mode conversion goes into electron heating.

A'single-species ion-cyclotron resonant layer, as a rule, is oriented so that the poloidal field plays no role and the short wavelength waves are desćribed by dispersion relation (51) and Fig. 8. According to (52-53), these waves are absorbed by electron Landau damping resulting in electron i híèting.

The next question which arises is: How strnng ic the absulpilon caused by the resonant layers? The W.K.B. dispersion relation do not ainswer this question, and a full wave solution must be used. The easiest way to do this is via the Budden model where the frequency is interpreted to be a Laplace transform variable having a small positive imaginary part which prevents the breakdown of the ideal equations. In the sprtion entitlcd "Ful1 Wave Calculations of Mode Conversion," we prove that full wave calculations based on dispersion relations (40) and (49) provide the same solutions to the ideal branches as does the Rudden model, justifying the use of a Budden model to compute the absorption of energy from the ideal branches. The derivation of a Budden equation from differential equations for wave propagations (76-77) was done in equations (78-82). Th1s Budden equation was used to estimate the damping of a toroidal eigenmode by the ion-ion hybrid resonance. The final result is formula (105) which shows that the ion-ion hybrid resonance effcctively prevents toroidal eigenmodes. In particular, the minority heatiug scheme proposed by stix [16] will not have high-Q eigenmodes.

A Budden-model calculation was also made of the damping decrement caused by a surface ion-cyclotron mode conversion layer. The Result (119-120) shows 
that the damping decrement caused by ion-cyclotron mode conversion is larger than the damping decrement resulting from fundamental resonant ion absorption (121). Consequently, fundamental ion-cyclotron heating experiments will lead predominantly to surface electron heating, as observed by Iiyoshi et al. [11]. Any ion heating will be inefficient - in agreement with experiments on the ST tokamak [17].

The predictions of the theory developed in this paper are in qua? itative agreement with the global parameters measured in several tokamak experiments: eigenmode Q's [1,2], ion heating efficiencies [1,3,17] and electron heating [11]. But the detailed physics of the mode conversion process remains unexamined experimentally. Basic research experiments are called for to investigate the nature and damping of the short wavelength waves, especially as a function of the poloidal field parameter $\varepsilon$ ' c.f. (35)].

Perhaps the most important question is: Is heating a tokamak via the ion-ion hybrid superior to heating via the ion second harmonic absorption or electron transit time damping of toroidal eigenmodes? The answer appears to be no. The ion-ion hybrid resonance suppresses the toroidal eigenmodes, which lowers the loading resistance of the launching coil [17]. High loading resistance results in technologically much simpler wave launching coils a very desirable feature from the reactor engineering point-of-view. Also second harmonic heating leads to efficient energy input into the bulk of the ion distribution - a feature required to make an ICRF-driven Tokamak Fusion Test Reactor,work well [20]. One should point out that true second harmonic heating of a tokamak plasma has yet to be carried out experimentally. In both the Princeton $[1,17]$ and Kurchatov [3] experiments, attempts to heat deuterons at the second harmonic were most likely contaminated by ion-ion 
hybrid effects caused by proton impurities. Experiments on TFR [2] at the second harmonic of the proton gyrofrequency, where there is no possibility of ion hybrid resonance effects, have shown the expected high-Q eigenmodes but have not yet had sufficient power to perform a heating experiment.

What are the possibilities of performing a true second harmonic heating experiment on the PLT tokamak with the planned $55 \mathrm{MHz}$ generator? The most interesting possibility is that of using $\mathrm{p}-\mathrm{He}^{3}$ plasmas and performing second harmonic heating of $\mathrm{He}^{3}$ at a magnetic field strength of $27 \mathrm{kG}$. One should note that a $\mathrm{p}-\mathrm{He}^{3}$ plasma is a precise model of a $\mathrm{D}-\mathrm{T}$ plasma in the ratio of gyrofrequencies. Since we wish to avoid ion-cyclotron mode conversion effects associated with the fundamental proton frequency, the geometry required for the second harmonic surface in the minor cross-section is that given by Fig. 15, which also applies to an ICRF-driven D-T plasma reactor [20].

True second harmonic heating of deuterium can proceed only if there is no contamination by proton ion hybrid effects which requires proton concentration $n_{p}$ sufficiently small so that

$n_{p}<\frac{n}{2 R}\left(\frac{2 T_{i}}{M_{p}}\right)^{1 / 2} \frac{1}{\Omega_{p}} \sim \frac{n}{200} T_{k e V}^{1 / 2}$

where $\mathrm{n}$ is the toroidal mode number. Even if (122) is satisfied, fundamental resonant absorption by protons will dominate second harmonic deuterium absorption provided $n_{P}>B_{i}$. Heating experiments which initially deposit the energy in a minority species comprising less than $1 \%$ of the particles can lead to a thermal runaway of the minority speciês. Hence the $\mathrm{p}-\mathrm{He}^{3}$ plasma appears to offer the most direct approach to second harmonic heating as well as being a good model for a $\mathrm{D}-\mathrm{T}$ reactor. 


\section{ACKNOWLEDGEMENTS}

Discussions with J. Adam, J. Hosea, J. Jaquinot, J. Scharer, D. G. Swanson, T. Stix and H. Takahashi have been truly valuable to my understanding of the ion-ion hybrid resonance. This work was partially supported by the U.S. Energy Research and Development Administration, Contract E(11-1)-3073.

\section{REFERENCES}

[1] TAKAHASHI, H., Phys. Rev. Lett. (submilted, 1977)

[2] T.F.R. GROUP, 6th Conf. on Plasma Physics and Controlled Nuclear Fusion Research, Berchetesgaden, FRG, paper IAEA. CN. 35/G8.

[3] VDOVIN, V.L., SHAPOTKOVSKII, N.V., RUSANOV, V.D., Third International Meeting on Theoretical and Experimental Aspects of Heating Toroidal Plasmas, Vol. 2, Invited and Post Deadline Papers, (Grenoble) 1976.

[4] PERKINS, F.W., CHANCE, M., KINDEL, J.M., Third Int. Symposium on Toroidal Confinement, Garching, 1973.

[5] KI.T.MA, R., Nuclear Fusion 11 (1.971) 357.

[6] BERGER, R.L., KULSRUD, R.M., PERKIiNS, F.W., Proceedings of Annual Meeting on Theoretical Aspects of Controlled Thermonuclear Research, Rosslyn, Va. (1975), paper D1.

[7] SinANSON, D.G., Phys. Rev. Lett. 36 (1976) 316.

[8] BUDDEN, K.G., Radio Waves in the Ionosphere, Cambridge University Press, Cambridge (1961) p. 476.

[9] BEKEFI, G.; Radiative Processes in Plasmas, John Wiley, New York (1966) p. 229.

[10] Swanson, D.G., Phys. Fluids 10 (1967) 428.

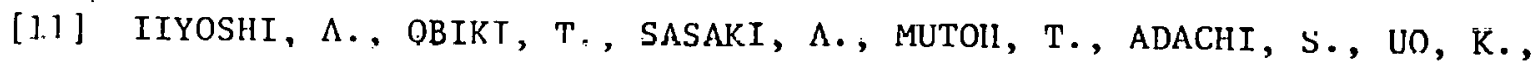
Third International Meeting on Theoretical and Experimental Aspects of Heating of Toroidal Plasmas, Vol. 2, Invited and Post Deadline Papers, Grenoble, 1976 . 
[12] CHEv, L., HASAGawa, A., Phys. Fluids $\underline{17}$ (1974) 1399. HASAgawA A., CHEN L., Phys. Rev. Letters 35 (1975) 370.

[13] TATARONIS, J.A., J. Plasma Physics 13 91975) 87.

[14] LANDAU, L.D., LIfSHITZ, E.M., Quantum Mechanics, Nonrelativistic Theory, Addison-Wesley, Reading Mass. (1958) p. 488.

[15] SWANSON, D.G., Third International Meeting on Theoretical and Experimental Aspects of Heating of Toroidal Plasmas, Vol. 1, Grenoble (1976) 109.

[16] STIX, T.H., Nuclear Fusion 15 (1975) 737.

[17] ADAM, J., CHAiNCE, M., EUBANK, H., GETTY, W., HINNOV, E., HOOKE, W.M., HOSEA, J., JOBES, F., PERKINS, F., SINCLAIR, R., SPERLING, J., TAKAHASHI, H., 5th Conf. on Plasma Physics and Controlled Nuclear Fusion Kesearch, I'okyo, 1974 , paper IAEA-CN-33/A9-5.

[18] HOSEA, J.C., SINCLAIR, R.M., Phys. Fluids 16 (1973) 1268.

[19] STIX, T.H., Phys. Rev. Lett. 15 (1965) 878.

[20] PERKINS, F.W., Third International Meeting on Theoretical and Experimental Aspects of Heating of Toroidal Plasmas, Vol. 2, Invited and Post-Deadline Papers, Grenoble, 1976.

[21] CAT.J.EN, J.D., Comments on Plasma Physics and Controlled Fusion 2 (1976) 159. 
TABLE I.

Nominal Parameters of the ATC and PLT

Ion-Cyclotron Heating Experiments

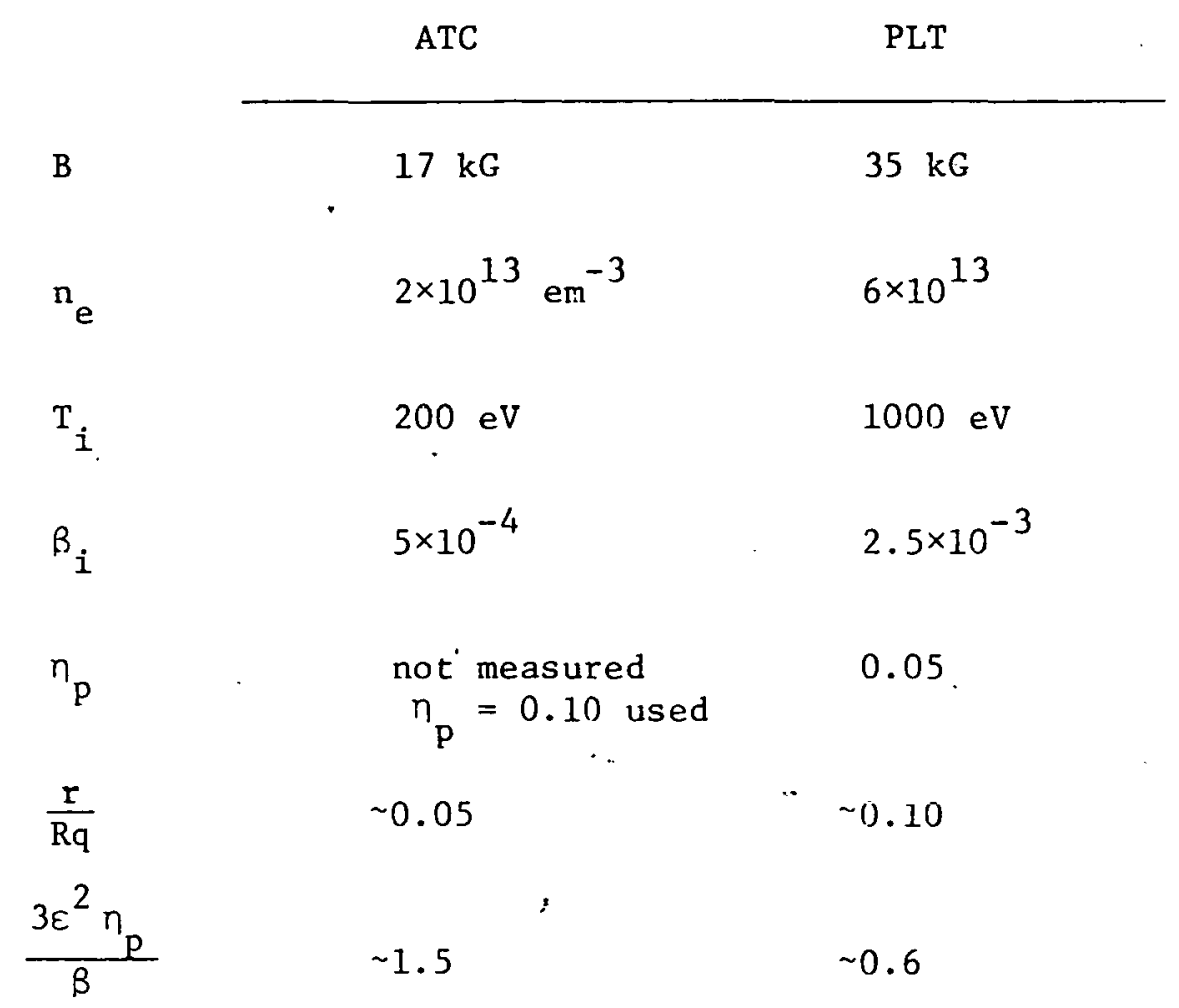




\section{Figure Captions}

FIG. 1. The nondimensional functions $\mathrm{K}$ corresponding to the functions $\mathrm{A}, \mathrm{B}$, $\mathrm{C}$ of equations (5-7). $\mathrm{K}=\mathrm{c}^{2} \mathrm{~A} / \omega_{\mathrm{pD}}^{2}$, etc. In the region $1.0<\mathrm{y}<1.5$, the $\mathrm{B}$ curve is off-scale and negative.

FIG. 2. Mode conversion resonances and cutoffs for a $95 \%$ deuterium plasma with a $5 \%$ proton concentration. (a) Curve $\dot{A}$ is the mode conversion resonance while curve $B$ gives the associated cutoff. Curve $C$ is the fast magnetosonic cutoff. The nondimensional density $N$ san bo oonverted to the duludl densicy by formula (13). (b) Sketch of the resonance and cutoff surfaces on a tokamak minor cross-section. The quantity $\delta a_{A}$ denotes the distance between the mode conversion resonance and the proton ion-cyclotron surface.

FIG. 3. Cutoffs and resonances in a plasma composed of $95 \%$ deuteri.1.m and $5 \%$ tritium. (a) Curve $A$ is the mode conversion resonance while curve $B$ is the associated cutoff. Curve $\mathrm{C}$ is the magnetosonic wave cutoff. (b) Geometry of curves in a tokamak minor cross-section for aspect ratios small enough to have both the deuterium and tritium cyclotron resonances in the plașa. (c) Geometry of curves when only the tritium cyclotron resonance is in the plasma.

FIG. 4. Cutoffs and resonances for a plasma composed of $50 \%$ deuterium and $50 \%$ tritium. (a) Curve $A$ is the mode conversion resonance while curve $B$ is the associated cutoff. Curve $\mathrm{C}$ is the magnetosonic wave cutoff. (b) Geometry of curves in a tokamak minor cross-section when both deuterium and tritium resonances are in the plasma. (c) Geometry of curves when only the deuterium resonance is in the plasma. 
FIG. 5. One dimensional model for a mode conversion layer. The x-direction is normal to the cutoff (B) and resonance (A) surfaces. The variation of the equilibrium in the $x$-direction can be due to either major radius variation in magnetic field or minor radius variation in plasma density depending on whether ion-ion hybrid or ion-cyclotron mode conversion processes are being considered. The angle $\theta$ between the poloidal field $\vec{B}_{p}$ and the $x$-direction depends on the orientation of the mode conversion surfaces (consult Fi $: 5: 2-4$ ).

FIG. 6. Mode conversion diagrams for a plasma composed of minority protons in a deuterium plasma. (a) Limit a where $\beta_{D} / 3 \varepsilon^{2} \eta_{p}=\delta_{a} \ll 1$. (b) Limit b where , $3 \varepsilon^{2} n_{p} / \beta_{D}=\delta_{b} \ll 1$. The notation $4-2$, etc. refers to a branch of the dispersion relation where terms in $\mathrm{s}^{4}$ are balancing those in $\mathrm{s}^{2}$. The hatched region shows the magnitude of $\mathrm{S}^{2}$ in regions where it is imaginary.

FIG. 7. Mode conversion diagram for a deuterium plasma containing minority tritium ions. The symbols A, B, C refer to the various branches of the dispersion relation referred to in the text. (a) Complete mode conversion diagram based on dispersion relation (48). (b) Simplified diagram based on dispersion relation (49). When $3^{2} \cdot e^{-1}$, then $k_{\perp}^{2} \mathrm{~T} / \mathrm{M} \Omega^{2} \sim 1$, and our FLR treatment breaks down.

FIG. 8. Ion-cyclotron mode conversion diagram for a single-species deuterium plasma. The notation 4-2, etc. refers to branches of the dispersion relation obtained by retaining only the $s^{4}, s^{2}$, etc. terms in dispersion relation (51). In the shaded portion where $s^{2}>\beta^{-1}$, our finite Larmor radius expansion breaks down. 
FIG. 9. Contours for solutions to the Budden equation. (54). The heavy lines correspond to branch cuts emanating from $k= \pm 1$. The contour $\mathrm{C}_{2}$ corresponds to the solution of (54) which is regular at the origin.

FIG. 10. Contours and branch cuts for the integral (64). (a) The contour and cuts corresponding to the solution of the Budden equation which is regular at the origin. (b) Contour corresponding to solution $\psi_{1}$ of equation (58). The open circles and crosses correspond to saddle points when $\xi>1$ and $\xi \ll-1$ respectively. The double arrow segments indicate the appropriate end points for contours according to (67).

FIG. 11. Contours and branch cuts for the integral (70). (a) The contour and cuts corresponding to the solution of the Budden equation which is regular at the origin. (b) Contour corresponding to solution $\psi_{1}$ of equation'(58). The open circles and crosses designate saddle points for $\xi \gg 1$ and $\xi \ll-1$ respectively. The notation A, B identifics the various saddle points with the branches of dispersion relation (49) for $x / \eta_{T} R \gg 1$ as depicted in Fig. $7 b$. The double arrow segments indicate the appropriate end points for contours according to (71).

FIG. 12. Slab geometry model for calculating the effect of the ion-ion hybrid resonance on toroidal eigenmodes. A plasma of uniform density and composition occupies the region between two conducting boundaries. The magnitude of the magnetic field $B$ changes with $x$ to produce the ion-ion hybrid resonance $R$ cutoff co pair. 
FIG. 13. Sketch of resonant and cutoff surfaces in a single-species deuterium plasma. The surface $A$ is the ion-cyclotron mode conversion surface described by (11). The surface $B$ is the ion-cyclotron cutoff while $C$ denotes the magnetosonic cutoff surface given by (12).

FIG. 14. Mode1 plasma density profile used to estimate ion-cyclotron mode conversion absorption. The letters A, B, C denote the corresponding surfaces of Fig. 13. The model is a slab model with a uniform magnetic field such that $1>(\Omega-\omega) / \Omega>0$.

FIG. 15. Geometry of various cyclotron resonant surfaces for second harmonic heating of tritium in a deuterium-tritium plasma. This diagram also applies to second harmonic heating of $\mathrm{He}^{3}$ in a proton $-\mathrm{He}^{3}$ plasma. The major radius. $R$ scale is normalized to unity at the second harmonic resonance for tritium. The circles represent the limiter radius for tokamaks of the indicated aspect ratio R/a. They are drawn so that the fundamental deuterium resonance is just avoided, placing the resonant heating surface as far towards the outside as possible, as suggested by flux-conserving high- $\beta$ tokamak equilibiria [21]. 


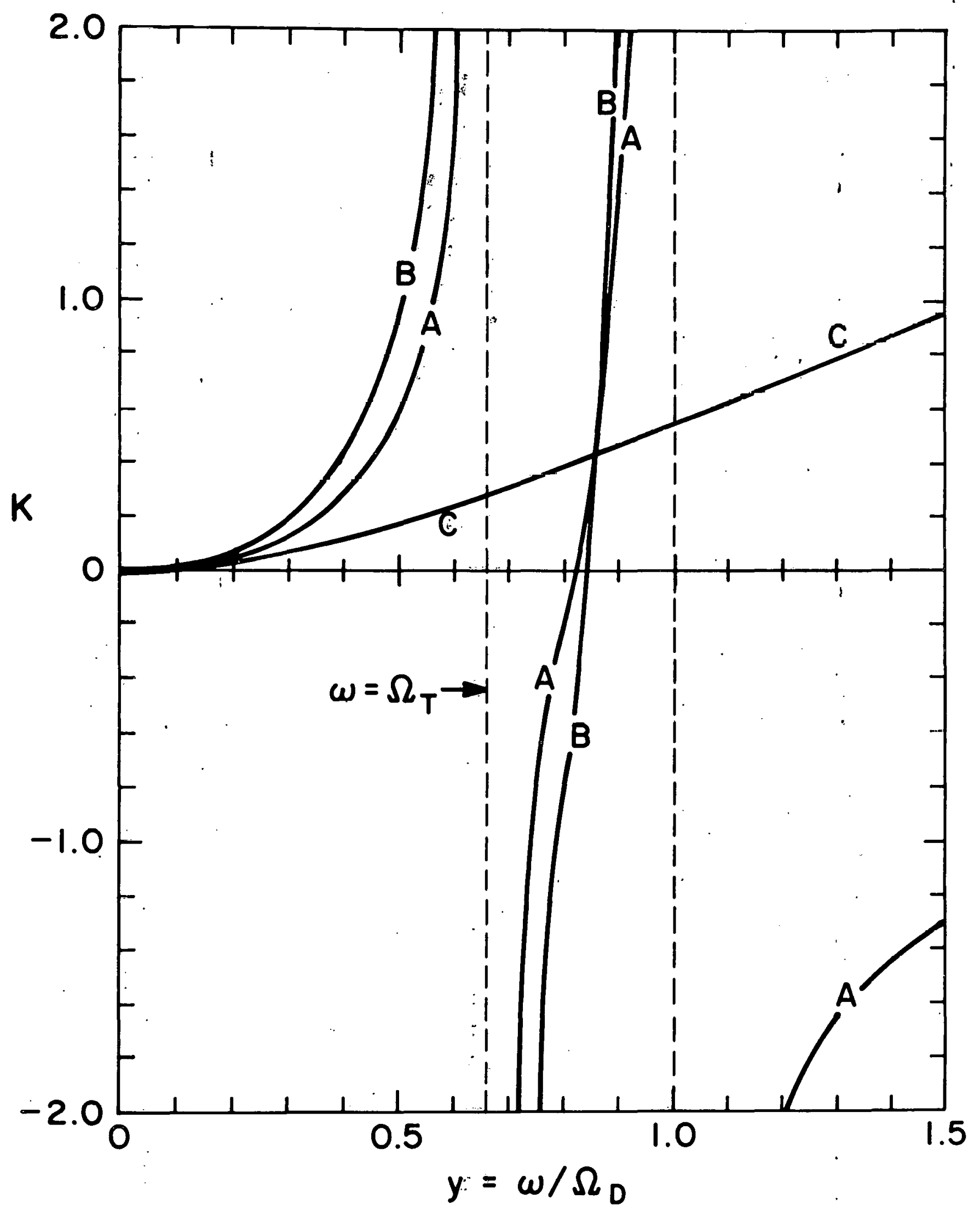

Fig. 1 . 


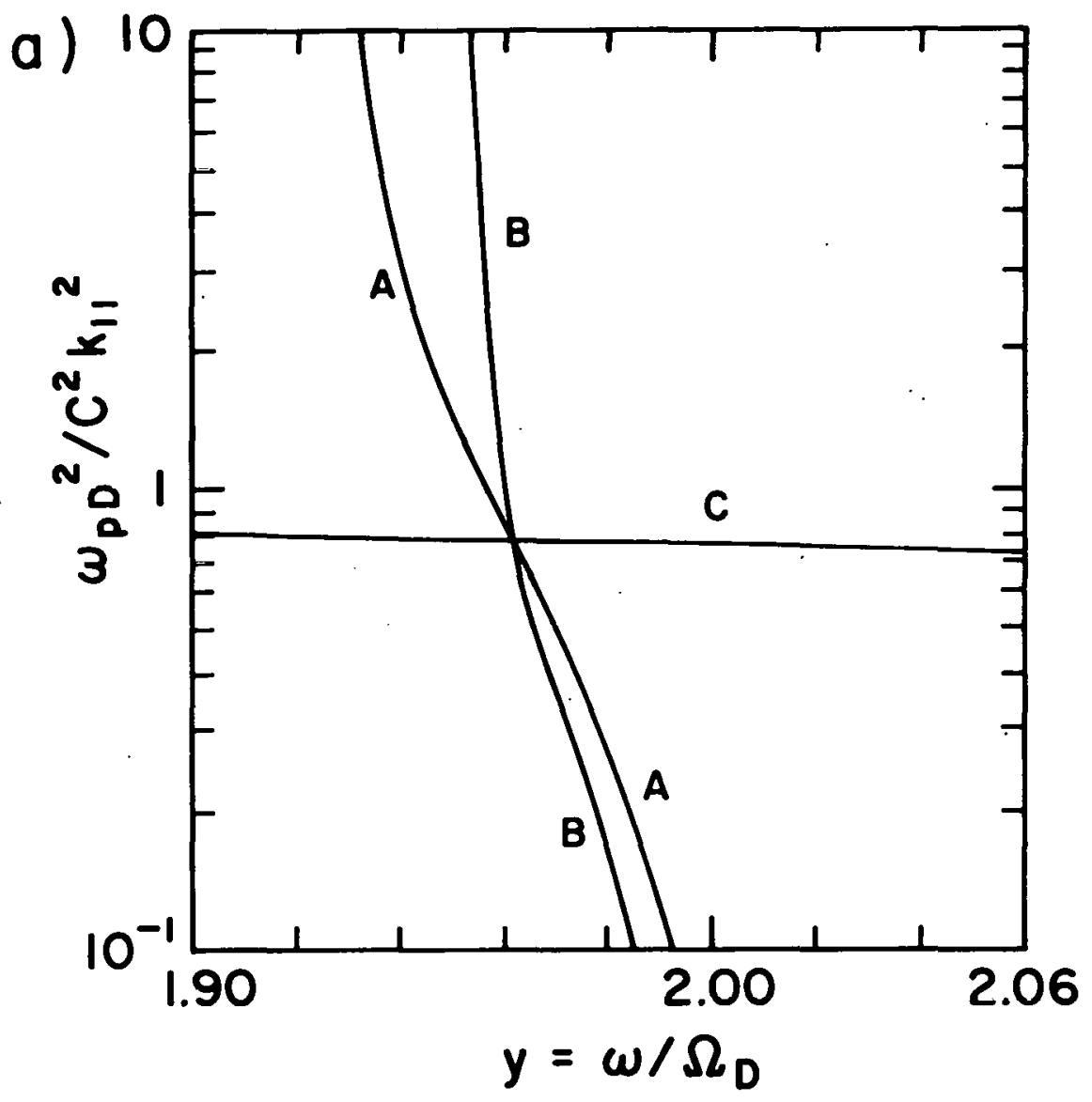

b)

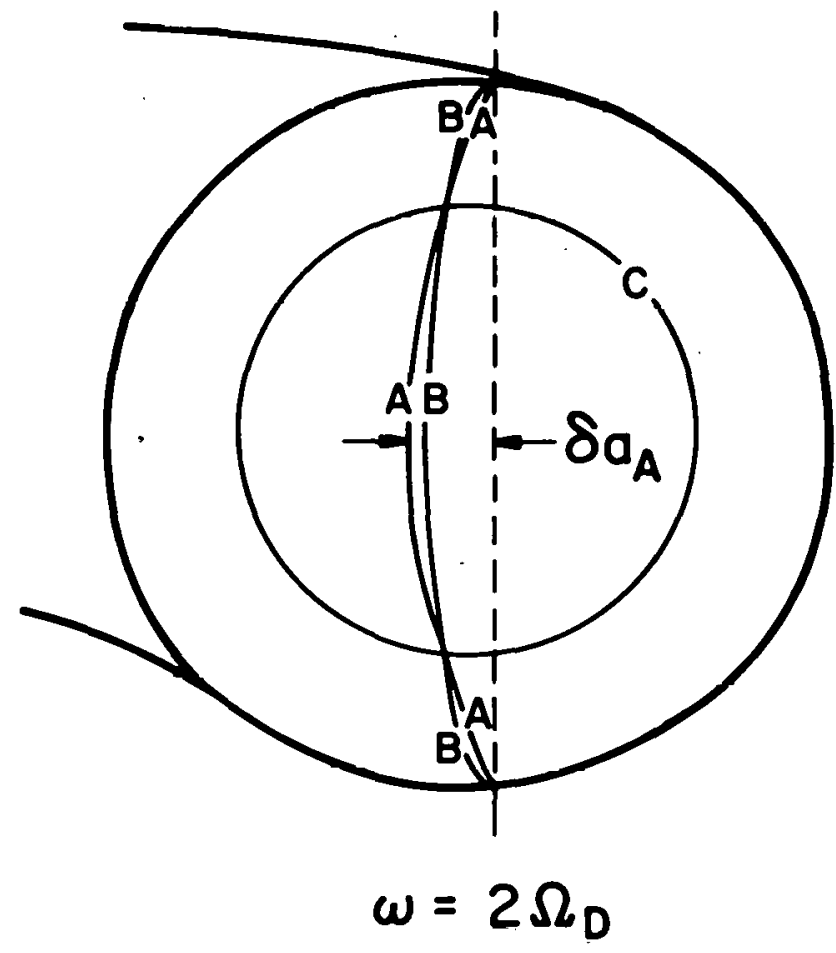

Fig. 2 . 

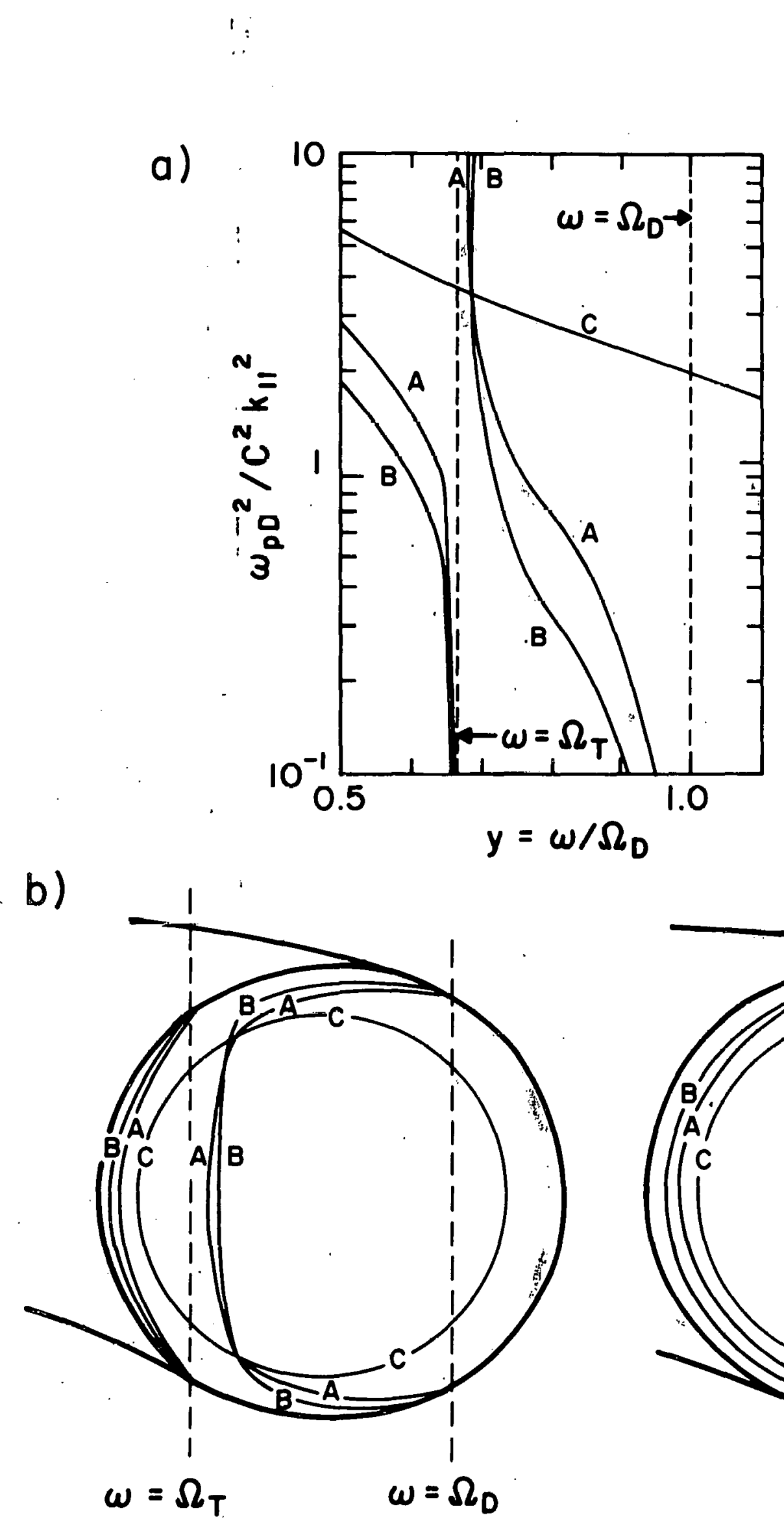

C) :

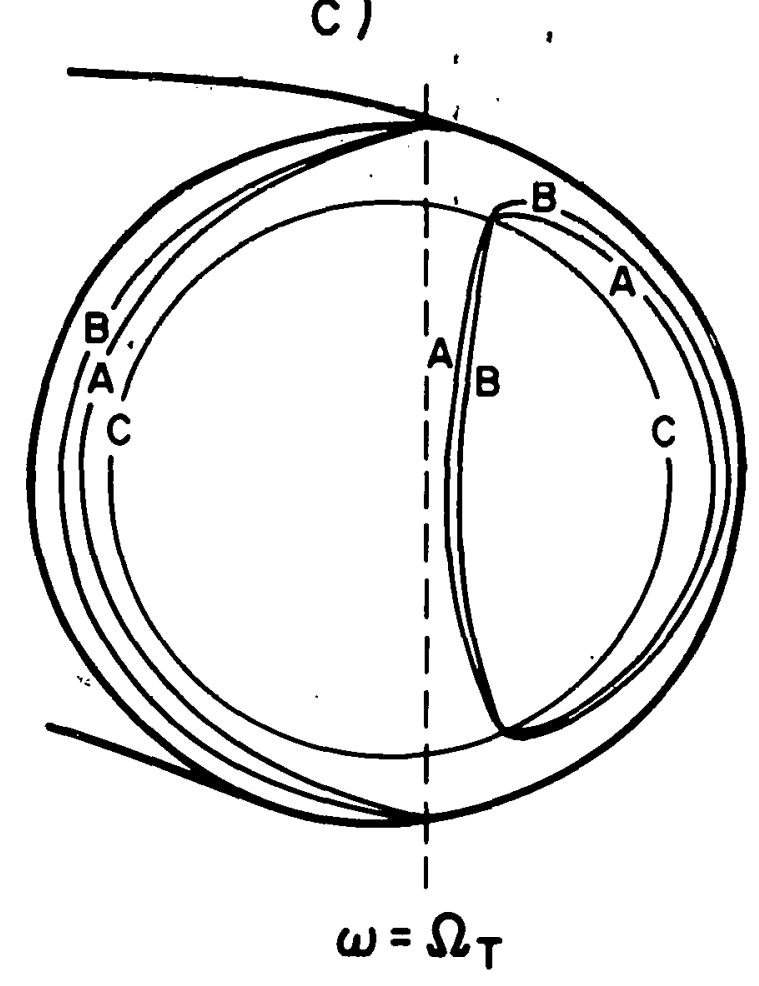

i.g. 3 


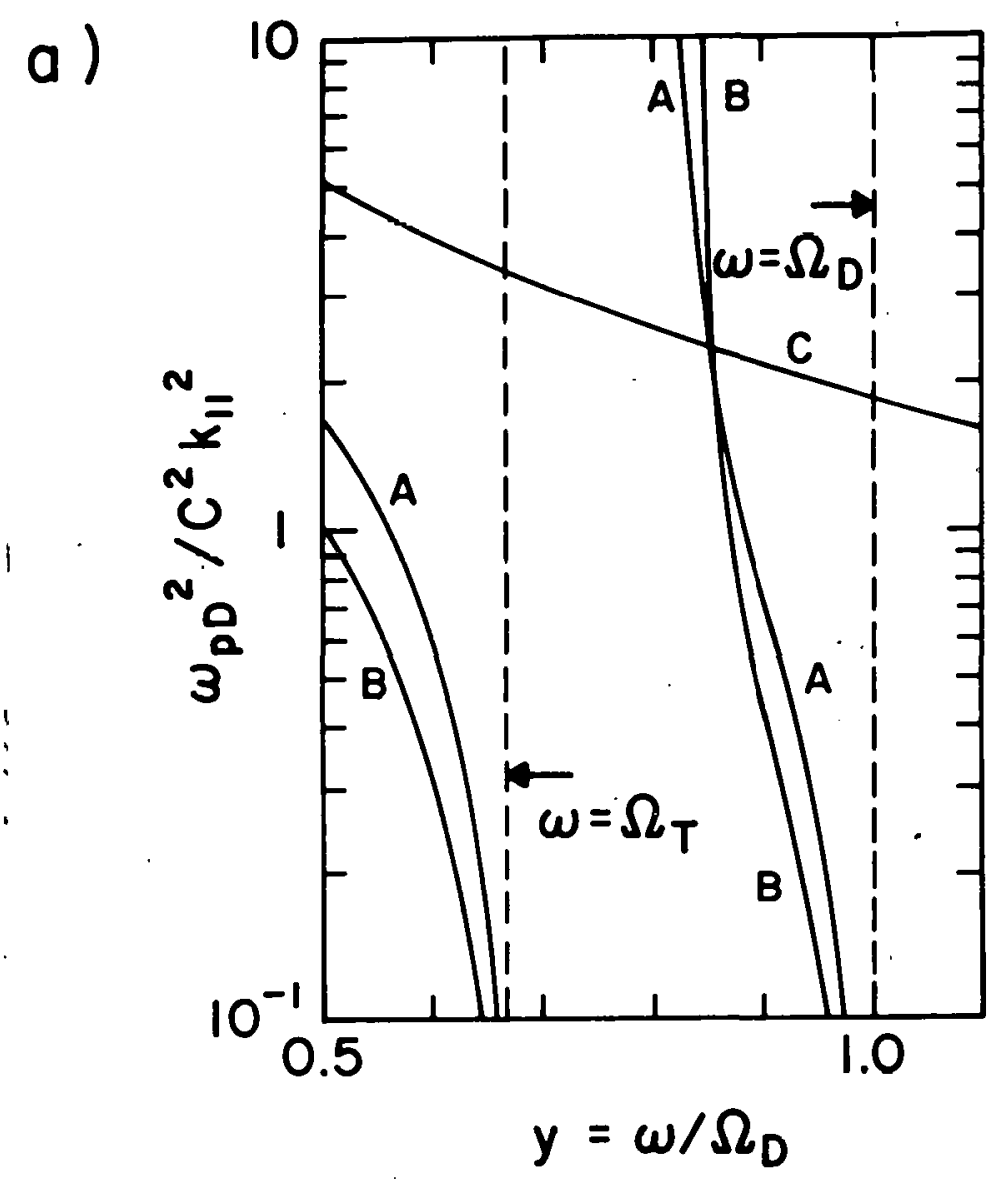

b)

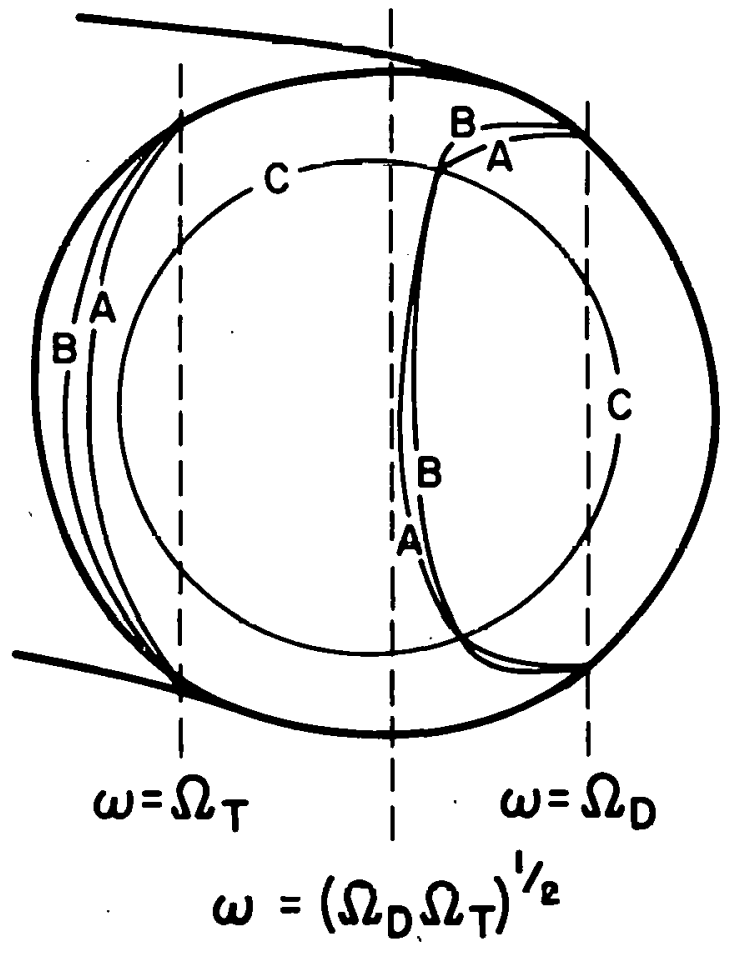

Fig. 4.

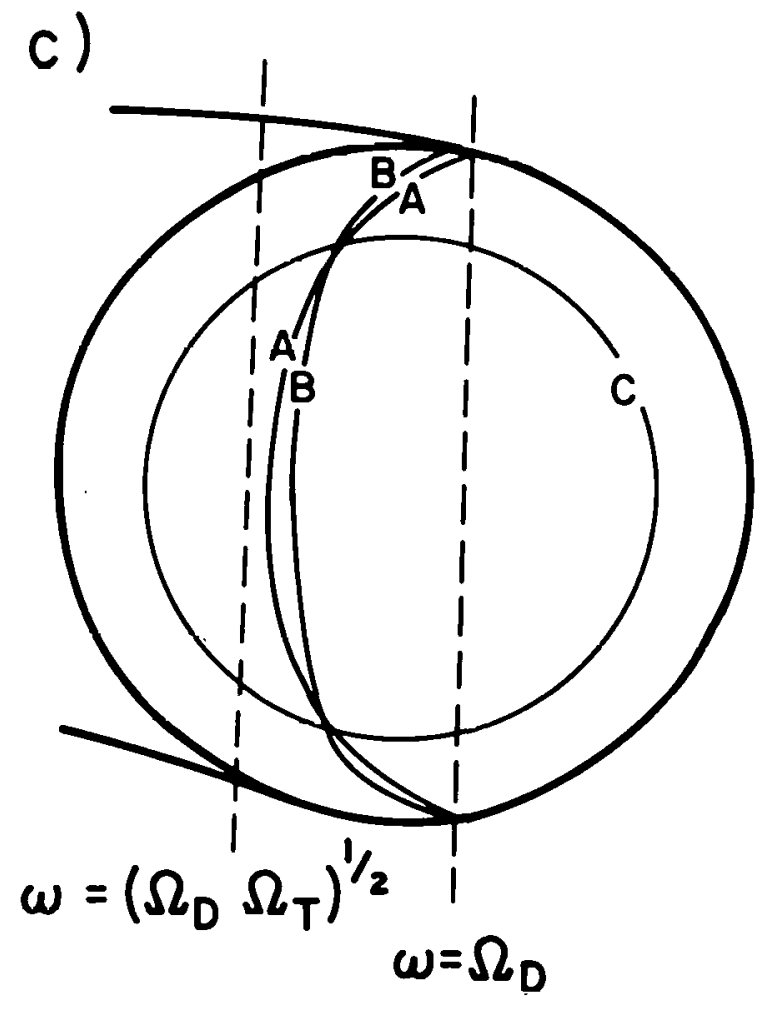




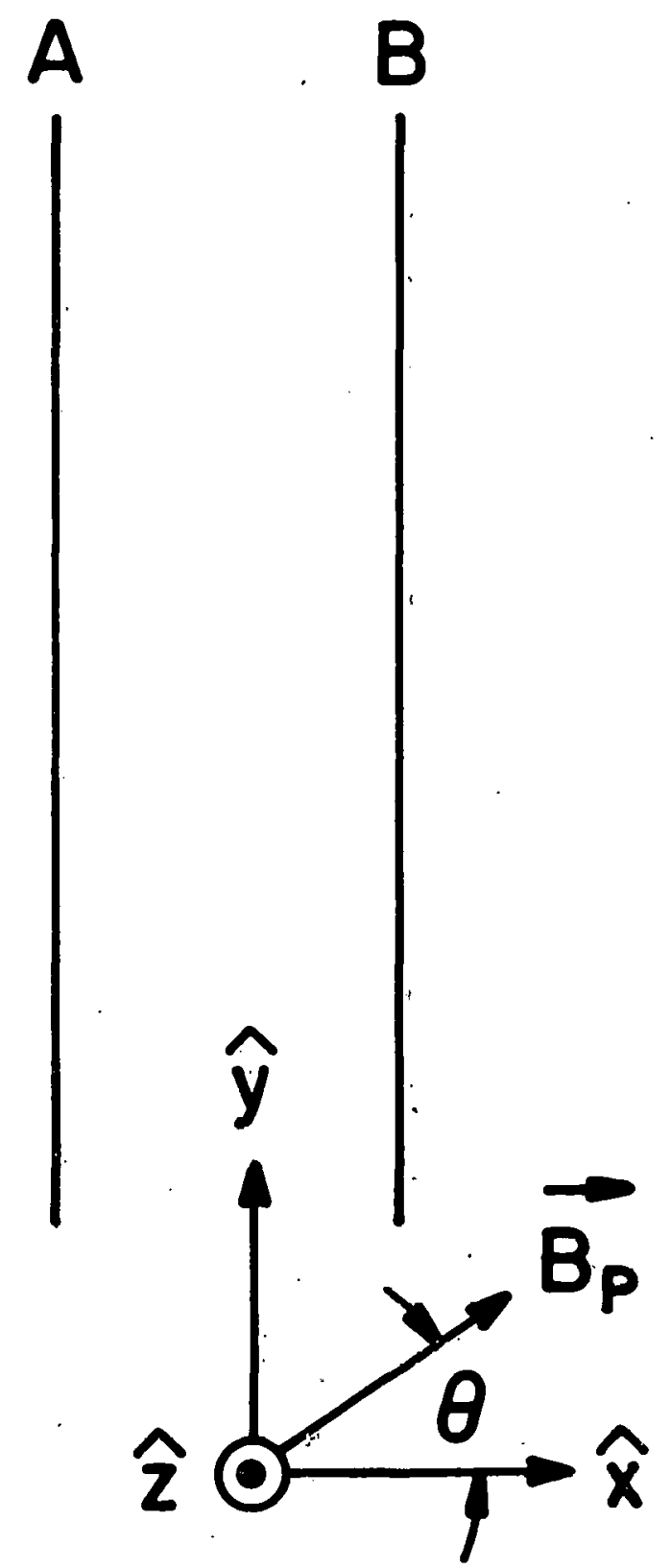

Fig. 5 . 

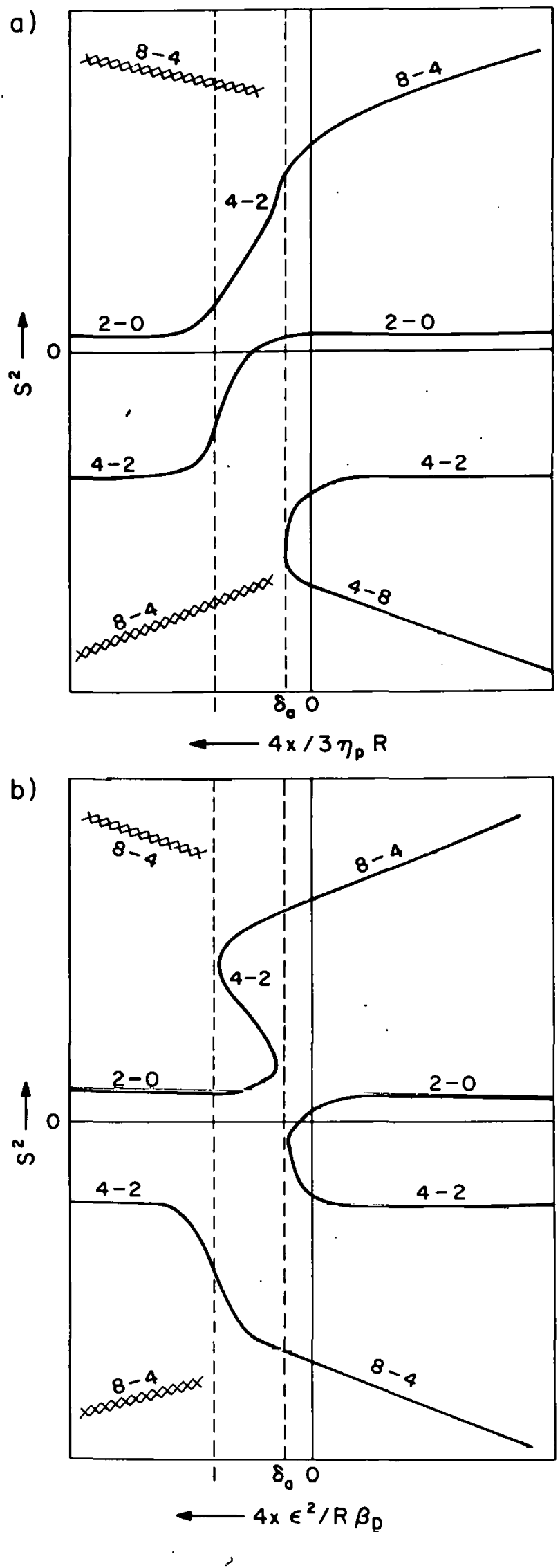

Fig. 6. 

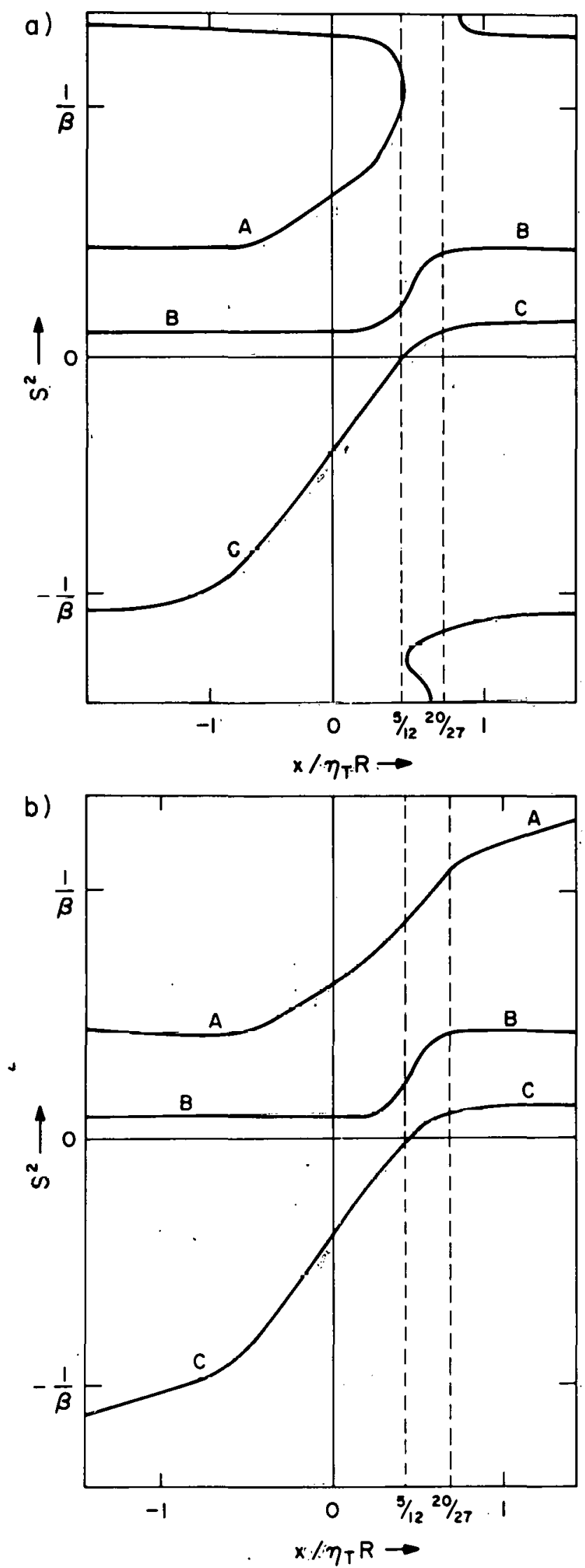

Fig. 7 . 


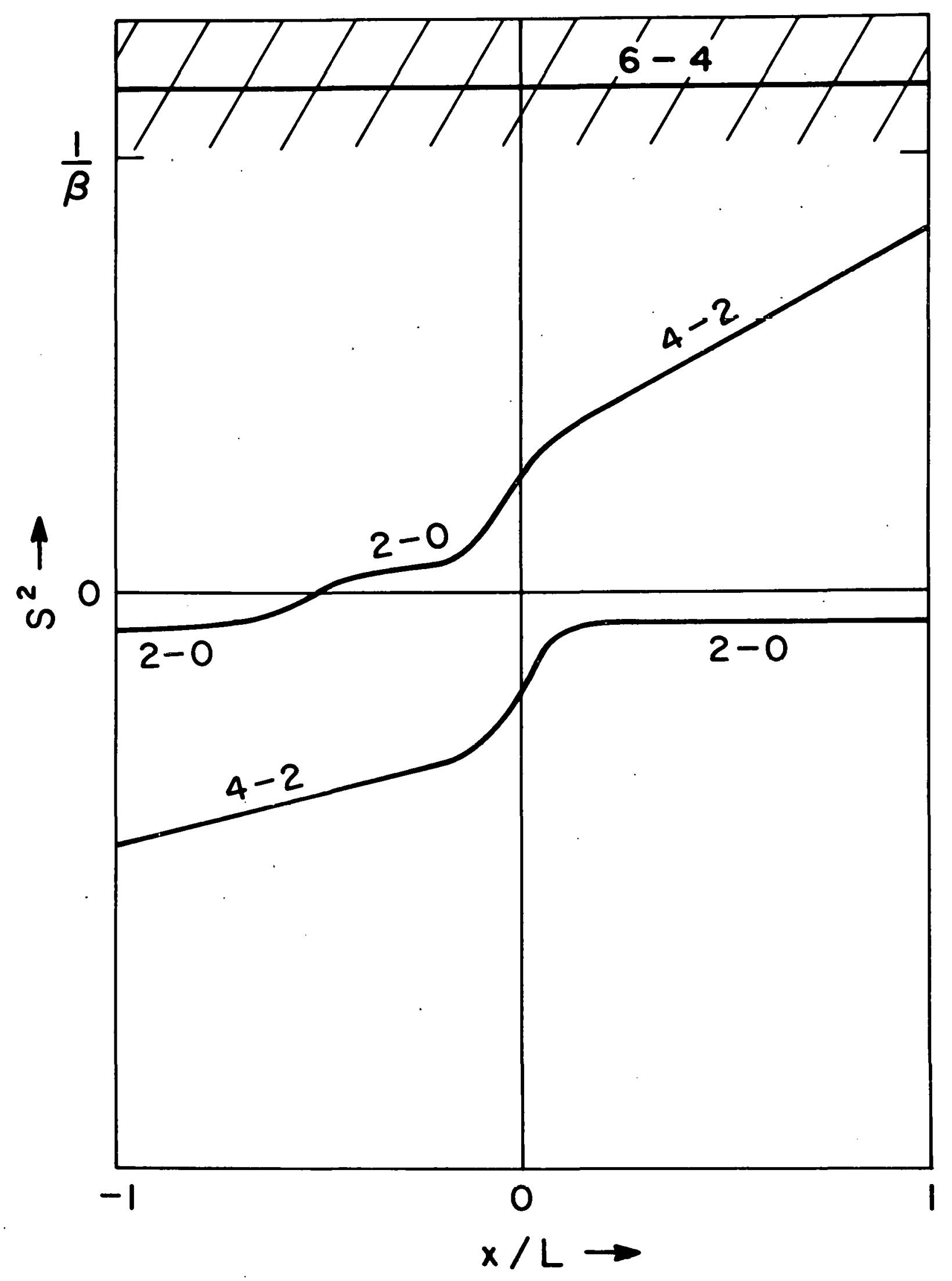

Fig. 8 . 

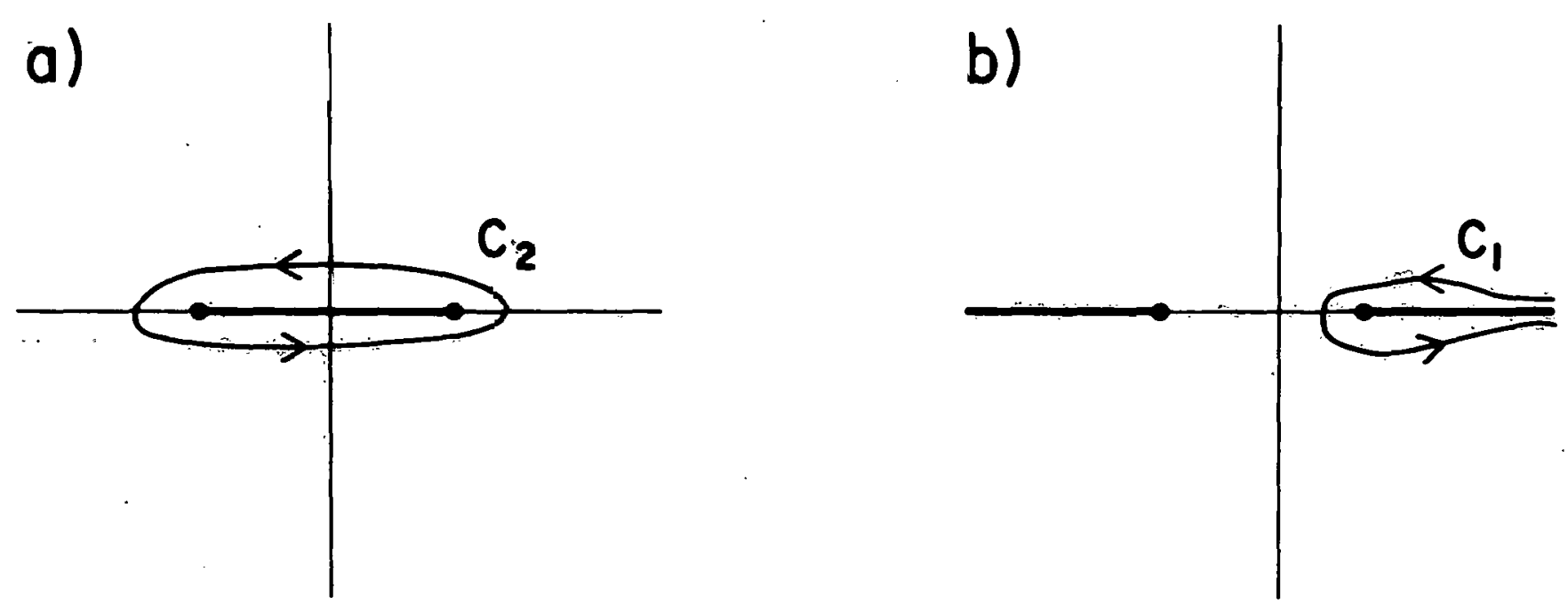

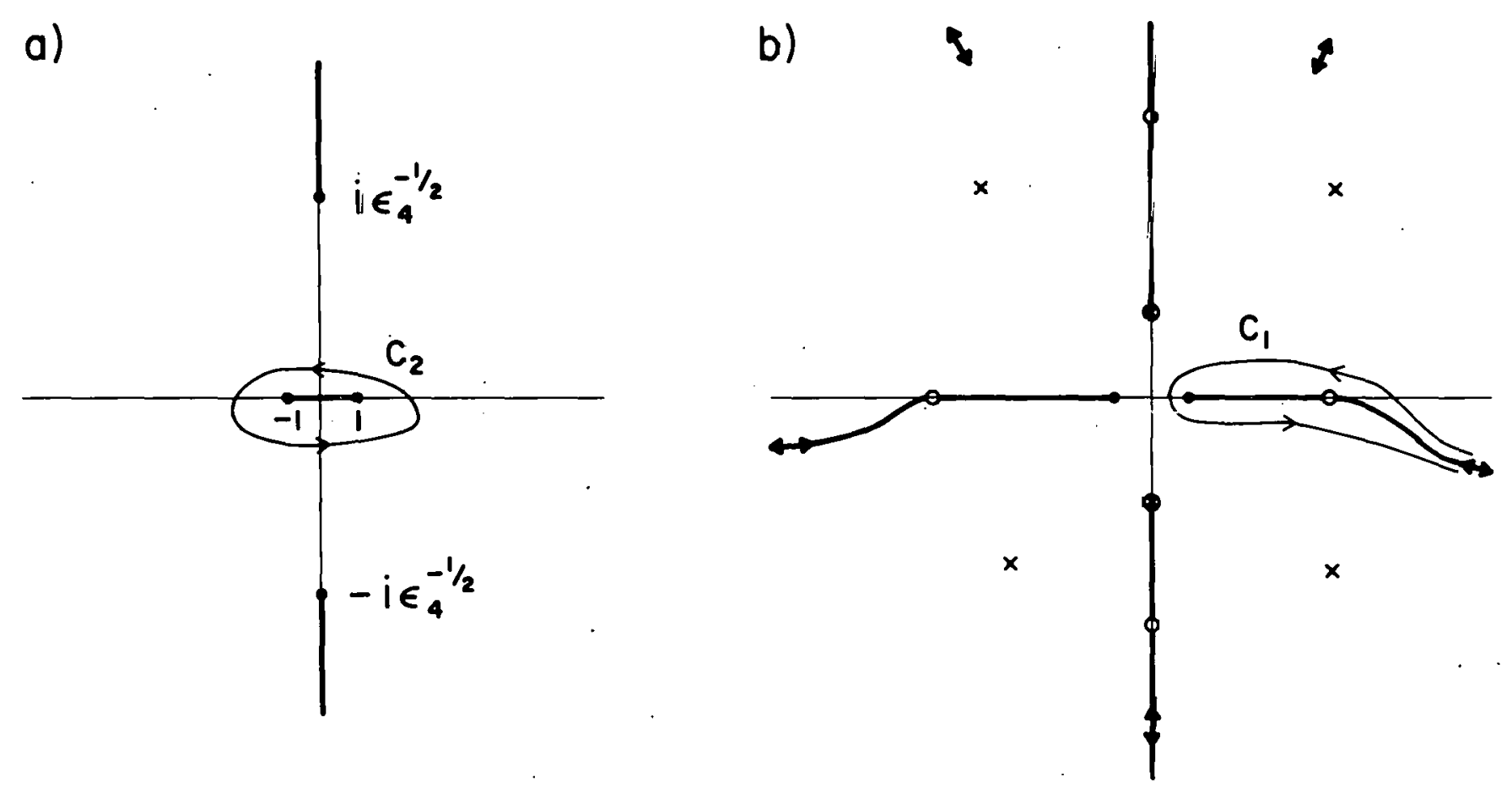
尔

a)

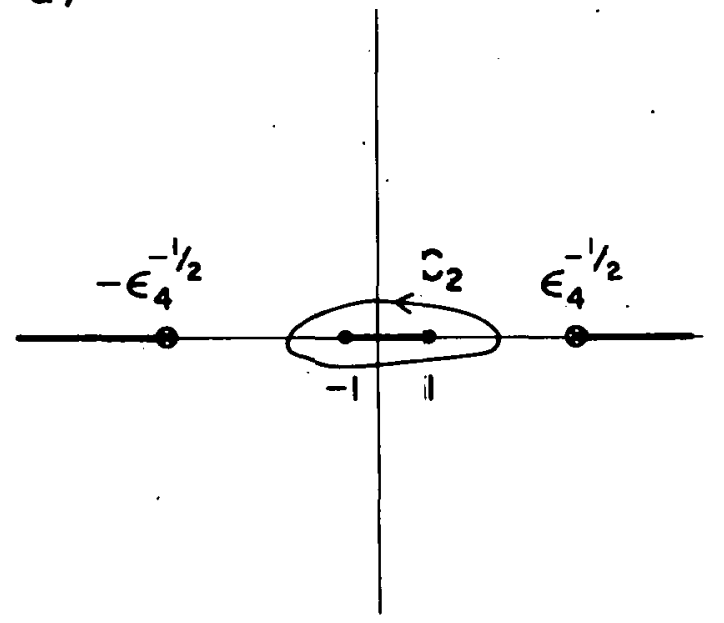

b) 


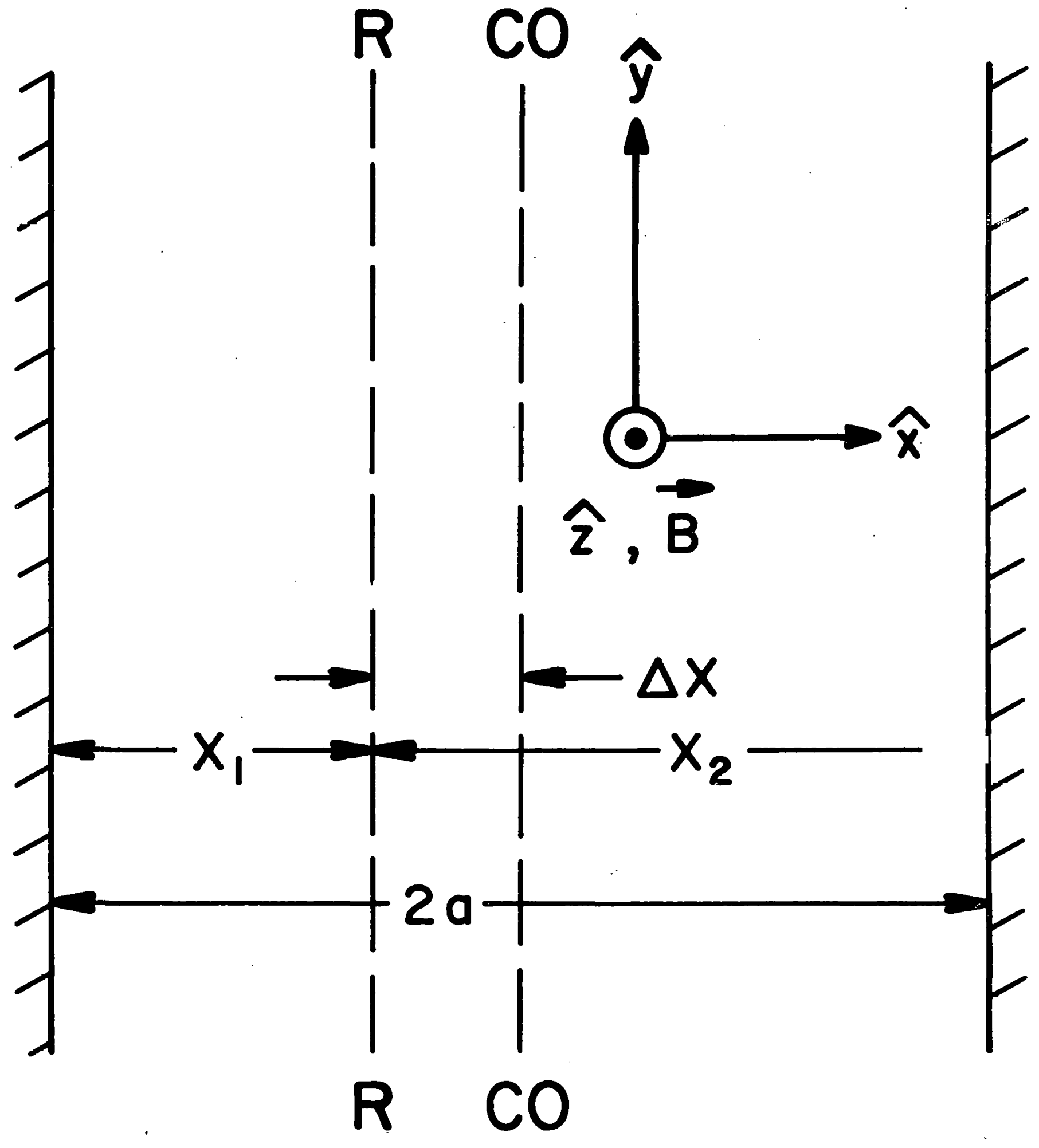

Fig. 12 . 


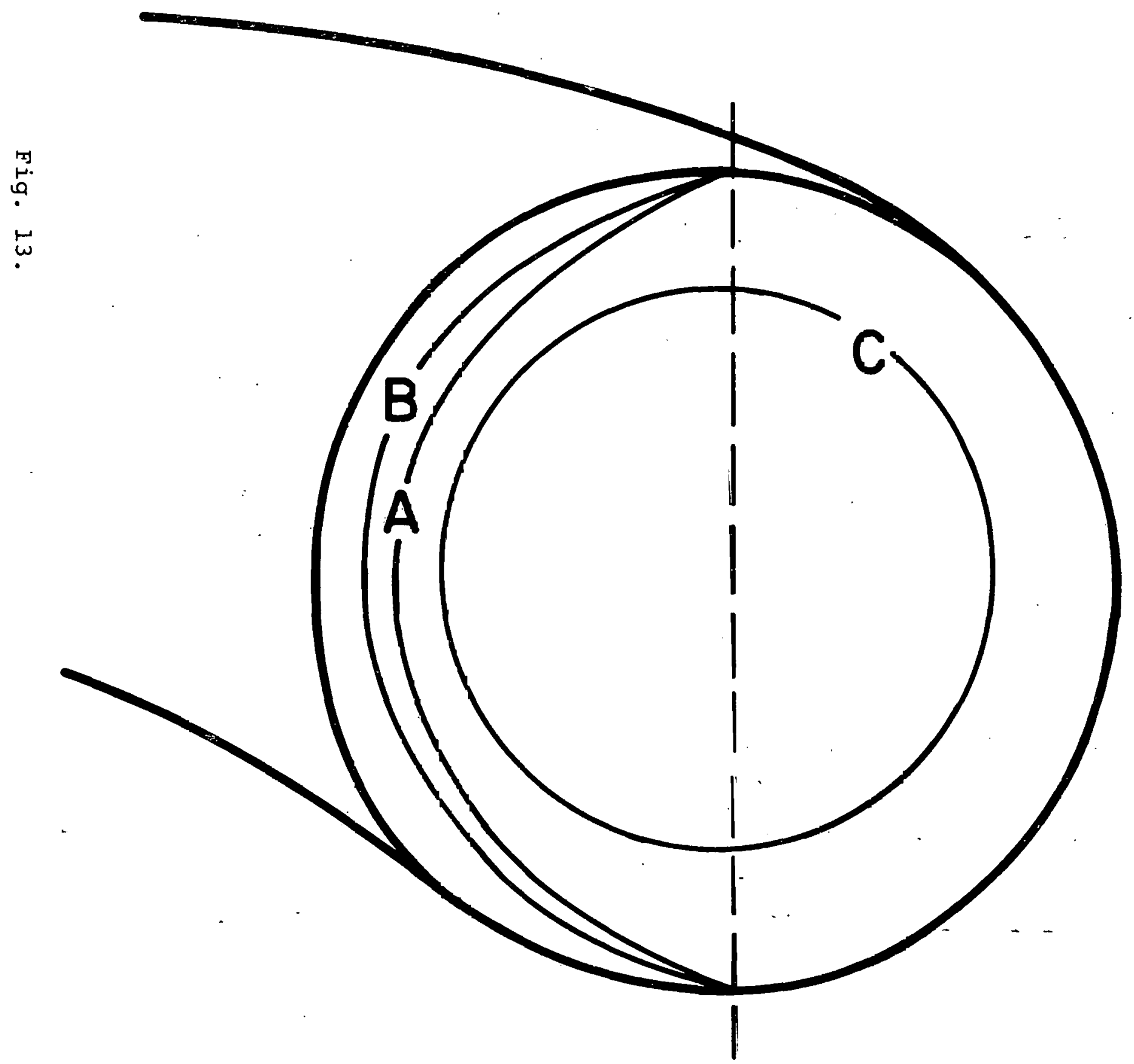




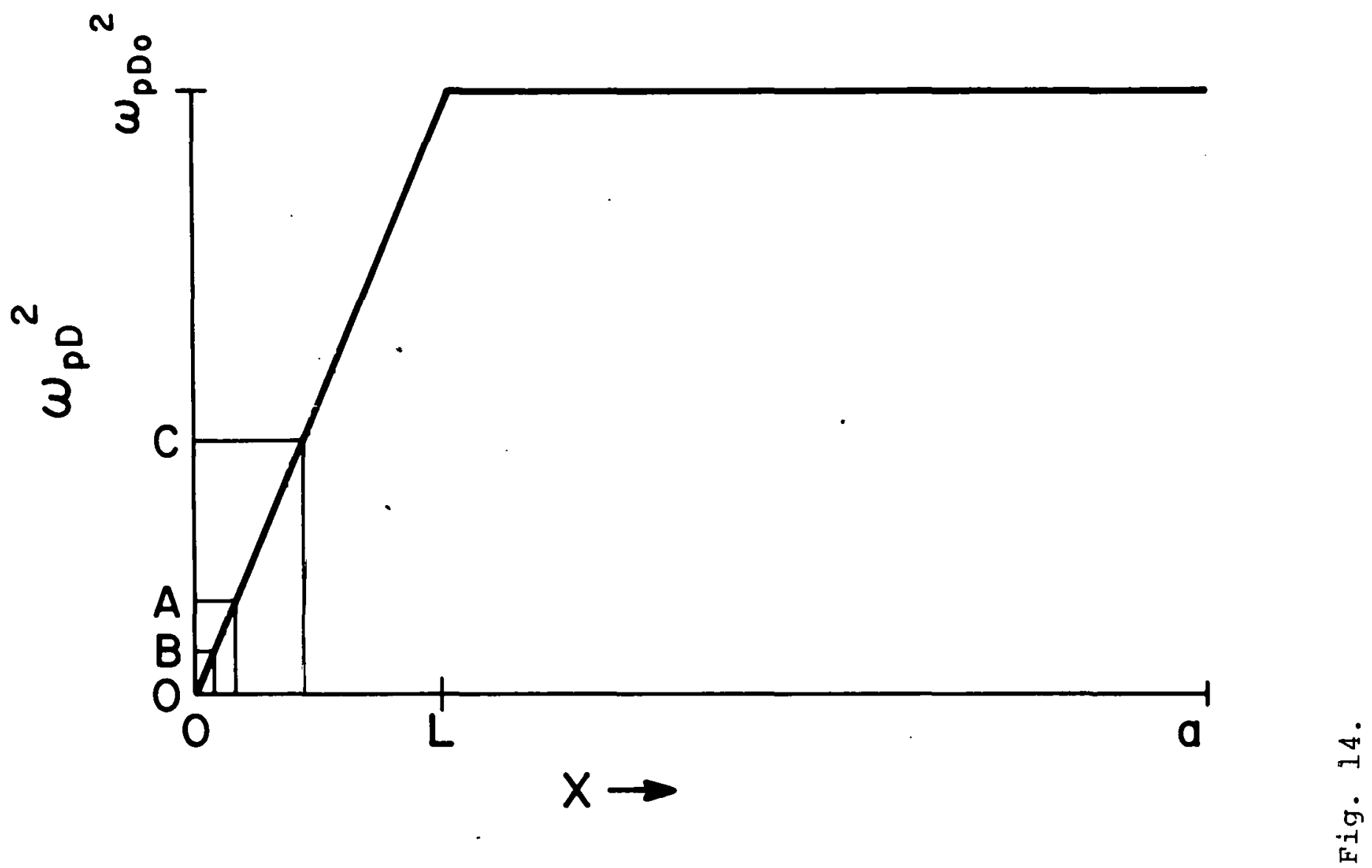




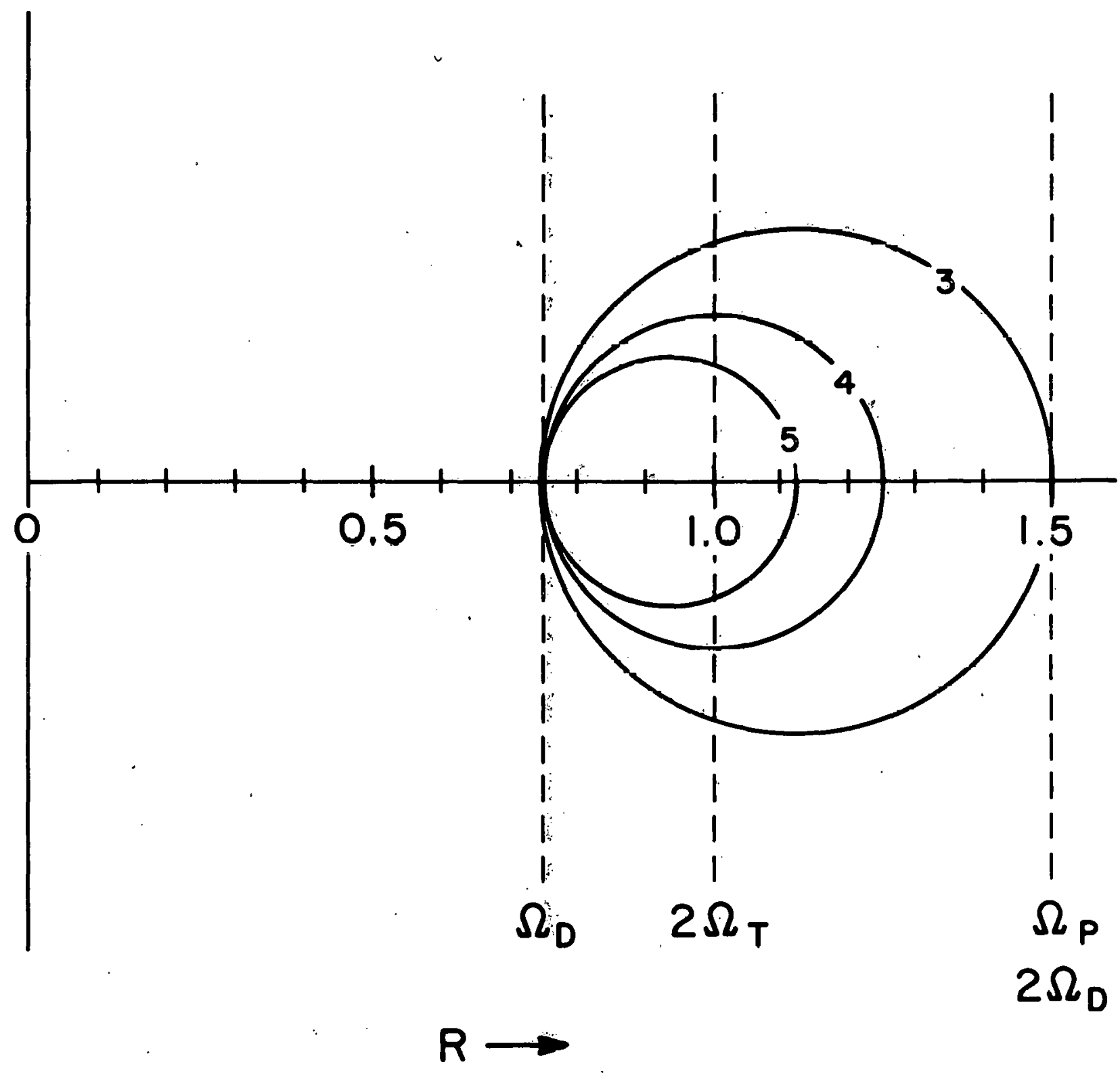

Fig. 15. 